\title{
REEVALUATING THE JET BREAKUP REGIME DIAGRAM
}

\author{
Ben Trettel*
}

\author{
University of Texas at Austin, TX 78712, USA \\ *Address all correspondence to: Ben Trettel, E-mail: http://trettel.us/contact.html \\ Original Manuscript Submitted: $m$ m/dd/2019; Final Draft Received: $m m / d d / 2020$
}

\begin{abstract}
Identifying the regime of a liquid jet is necessary to determine the physical mechanisms causing breakup and consequently how to model the jet. Existing regime diagrams are based on a small amount of data classified by superficial visual characteristics, making these diagrams too inaccurate to reliably determine the correct regime. A more accurate regime diagram is developed using primarily a large compilation of breakup length data combined with theory where the data is sparse. Improvements in the regime diagram include a new regime, the addition of two critical Reynolds numbers and the turbulence intensity as variables, and the recognition that how the regimes change with increasing velocity (i.e., Rayleigh to first wind-induced to second wind-induced to atomization) is not universal.
\end{abstract}

KEY WORDS: liquid jet breakup, regimes, turbulence intensity, dripping, Rayleigh regime, first wind-induced, second wind-induced, atomization, turbulence transition, critical Reynolds number

\section{INTRODUCTION}

Liquid jets break up through many mechanisms, and most mechanisms must be modeled differently. Even focusing solely on the case of the breakup of circular Newtonian jets injected into still low density ratio environments without considering cavitation, compressibility, evaporation, or combustion, there are many varieties of jet breakup. This is the case considered in this work* Which "regime" a jet is in depends on factors including but not limited to the Reynolds number, Weber number, the liquid-gas density ratio, and the turbulence intensity.

Accurately determining the regime is necessary for both research on and design of systems in liquid jet breakup. Engineers often apply models applicable only in a particular regime to an inappropriate regime. Researchers may decide they want to study a particular regime, and consult a regime diagram to determine where to place their study. Frequently, the study is placed in a regime different from that intended. Similarly, when an engineer designs a spray system, they may target a particular regime for its breakup properties. For example, in fuel sprays smaller droplet sizes and breakup lengths are advantageous, while in fire hoses and water jet cutting larger droplet sizes and breakup lengths are advantageous. If the description of the breakup in a particular regime is inaccurate, an engineer may target the wrong regime. And if the regime diagram is inaccurate, an engineer can target the correct regime but place the system in the

* A complementary study focusing on non-Newtonian viscoelastic jets was published nearly simultaneously with this one (Brenn and Stelter 2020). 
wrong regime. All of these problems are avoided through a more accurate regime diagram and understanding of each regime.

Conventionally, the regime of a liquid jet has been determined through qualitative comparison of the appearance of the jet against prototypical jet images in the literature, e.g., Lin and Reitz (1998, fig. 1). Classification of images has also been used to produce regime diagrams which allow determination of the regime given variables like the jet Reynolds and Ohnesorge numbers. Unfortunately jets in several regimes appear superficially similar (e.g., the "second wind-induced" and "atomization" regimes), despite differing in terms of quantitative characteristics like the trend in the breakup length curve, as will be discussed. Frequently the superficial similarity of some regimes causes qualitative visual classification to be inaccurate. For this reason, quantitative characteristics are preferred when classifying regimes.

Regime diagrams are also typically constructed from relatively little data, lacking the resolution needed to precisely determine the boundaries of each regime. This problem is avoided in this work through a large compilation of data from the open literature and a small amount of new data ( $1.5 \%$ of the compilation) - see $\S 4.1$. Additionally, this data compilation specifically included only cases with known turbulence intensity, a measure of the strength of the turbulence, to be defined shortly. The influence of this variable on jet breakup in general is typically only hypothesized, and rarely validated against experimental data with appreciable turbulence intensity variation.

In this paper I'll first discuss the most popular regime diagram as of this writing in $\S 2$, then detail problems with this regime diagram in $\S 3$ After that, in $\S 4 \mathrm{I}$ will discuss a largely new regime diagram which is much more accurate than any previous diagram for the cases of interest in this work. A reader interested only in the new regime diagram can skip to $\$ 4$ if desired.

The notation used in this work is identical to that of a related theory paper (Trettel, 2020a). The nozzle outlet is specified with the subscript 0 , e.g., $\bar{U}_{0}$ is the jet's bulk velocity and $d_{0}$ is the nozzle outlet diameter. The subscript $\ell$ refers to liquid properties, e.g., $\rho_{\ell}$ is the liquid density. Similarly, the subscript $\mathrm{g}$ refers to gas properties, e.g., $\rho_{\mathrm{g}}$ is the gas density. The liquid phase Weber number $\mathrm{We}_{\ell 0} \equiv \rho_{\ell} \bar{U}_{0}^{2} d_{0} / \sigma$ where $\sigma$ is the surface tension of the liquid. A gas phase Weber number can be defined as $\mathrm{We}_{\mathrm{g} 0} \equiv \rho_{\mathrm{g}} \bar{U}_{0}^{2} d_{0} / \sigma$. The liquid phase Reynolds number is $\operatorname{Re}_{\ell 0} \equiv \bar{U}_{0} d_{0} / v_{\ell}$ where $v_{\ell}$ is the liquid kinematic viscosity. The plane-averaged turbulent kinetic energy at the nozzle outlet (analogous to the bulk velocity) is $\bar{k}_{0}$. Using this particular definition of the turbulent kinetic energy, the turbulence intensity can be defined as $\overline{\mathrm{Tu}}_{0} \equiv\left(2 \bar{k}_{0} / 3\right)^{1 / 2} / \bar{U}_{0}$. The motivation behind this particular definition of the turbulence intensity is described by Trettel (2019, 2020a). This paper assumes a basic understanding of turbulent flows. The reader unfamiliar with turbulence is recommended to consult a textbook like Pope (2000).

There are multiple quantities of interest in jet breakup, however, this work focuses most heavily on the (average) breakup length $\left\langle x_{\mathrm{b}}\right\rangle$, a measure of the droplet size like the Sauter mean diameter $D_{32}$, the (average) breakup onset location $\left\langle x_{\mathrm{i}}\right\rangle$, and the spray angle $\theta_{\mathrm{i}}$. The breakup length, breakup onset location, and spray angle are shown schematically in figure 1 The breakup length is defined in this work as the average distance from the nozzle outlet to where the core of the jet ends. The instantaneous breakup length is $x_{\mathrm{b}}$, so the averaged breakup length is $\left\langle x_{\mathrm{b}}\right\rangle$ using angle brackets to indicate averaging. Multiple experimental techniques can obtain the breakup length consistent with this definition. This is demonstrated by the excellent fit $\left(R^{2}=0.958\right.$ for 193 data points) of regressions like equation 31, sourced from both electrical conductivity and quantitative imaging measurements of the average breakup length. Similar to the breakup 


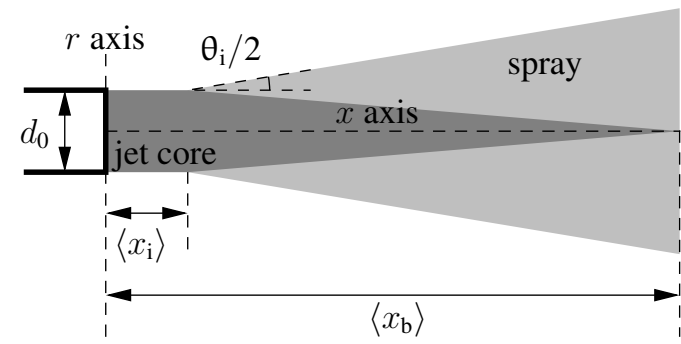

FIG. 1: Jet breakup variables labeled on a schematic liquid jet. $d_{0}$ is the nozzle outlet diameter, $\left\langle x_{\mathrm{i}}\right\rangle$ is the average breakup onset location, $\theta_{\mathrm{i}}$ is the spray angle, and $\left\langle x_{\mathrm{b}}\right\rangle$ is the breakup length.

length, the breakup onset location is defined as the average distance from the nozzle outlet to where breakup first starts.

\section{PREVIOUS REGIME DIAGRAMS}

The conventional regimes are (Birouk and Lekic, 2009, Lefebvre and McDonell, 2017, and Lin and Reitz, 1998):

1. Dripping regime - Breakup is driven by gravity, producing relatively large droplets.

2. Rayleigh regime - Breakup due to a surface-tension-driven instability resulting in droplets larger than the nozzle outlet diameter but of the same order of magnitude $\left(D \approx 1.89 d_{0}\right)$. The breakup length increases with increasing jet velocity in this regime.

3. First wind-induced regime - Convention states that the droplet diameters are on the order of the nozzle outlet diameter (Lin and Reitz, 1998, fig. 1), however, I challenge this view later in this paper — see the end of $\S 4.6$ Similarly, the breakup onset location is conventionally stated as many diameters from the orifice, but this is not necessarily true either. However, previous researchers did correctly understand that the breakup length decreases with increasing jet velocity in this regime (Reitz, 1978, p. 165, fig. 1.1).

4. Second wind-induced regime - The droplet diameters are smaller than the nozzle outlet diameter. The average breakup onset location is not negligible, but can be small. The breakup length increases following a power law with increasing jet velocity.

5. Atomization regime - The droplet diameters are much smaller than the nozzle outlet diameter. Frequently, in the atomization regime breakup is claimed to start at the nozzle outlet (i.e., $\left\langle x_{\mathrm{i}}\right\rangle=0$ ), though this is probably not true as will be discussed in $\S 3.8$. In this work I instead suggest that the breakup onset location is small. In the absence of cavitation and compressibility effects, the breakup length plateaus as the jet velocity is increased.

These regime names have slowly changed since the early works of Haenlein (1932) and von Ohnesorge (2019) (year of translation, published in German in 1936). The names for qualitative visual regimes and quantitative breakup length regimes can differ. For example, Lefebvre and McDonell (2017, figs. 2.9, 2.10, 2.13) have three different regime diagrams, two based on qualitative criteria with different regime names (one archaic, the other state-of-the-art), and another based on quantitative breakup length data with completely different regime names. The

Volume 30, Issue $x, 2020$ 
qualitative and quantitative regimes have been unified by some in the past so that a qualitative description of jets in a regime has an associated quantitative behavior (Reitz, 1978, p. 165, fig. 1.1). In this work, a unified regime diagram is proposed such that the quantitative and qualitative classifications are consistent, as shown in $\S 4.12$. Some regime names will also be changed to be more consistent with the physical mechanisms present in each regime.

Reitz (1978, pp. 4-9) has a detailed discussion of earlier research into the boundaries of the breakup regimes, which for the most part remains current - Reitz's work continues to be cited by more recent reviews (Birouk and Lekic, 2009; Chigier and Reitz, 1996, Lefebvre and McDonel1, 2017, and Lin and Reitz, 1998). Several criteria have been proposed, to be discussed shortly. These criteria have not been treated as unimpeachable, but they are treated as accurate enough to use for the planning of experiments in a particular regime.

A summary of selected past regime studies is in table 1 The first regime diagram was due to von Ohnesorge (2019) in 1936 and is reproduced in figure 2 By computing the nozzle Reynolds number $\mathrm{Re}_{\ell 0}$ and also what today is called the Ohnesorge number $\mathrm{Oh}_{\ell 0} \equiv \mu_{\ell} /\left(\rho_{\ell} \sigma d_{0}\right)$., an engineer can determine the regime by the location of the point in figure 2 (with some caveats; the nozzle and liquid-gas density ratio need to be similar to von Ohnesorge's). While some modifications to von Ohnesorge's diagram have been made since 1936, nearly the same regime diagram is used today as can be seen in the recent book of Lefebvre and McDonell (2017. figs. 2.9, 2.10).

The Ohnesorge number is independent of the velocity, which means that in these coordinates, increasing the velocity only changes the Reynolds number. Alternative coordinates (including the one I propose) lack this property, but the advantages gained from switching coordinates outweigh this small benefit. Regime boundary equations are typically constant gas or liquid Weber numbers (as physical arguments often suggest), so it would be simpler to create a plot in terms of the Weber and Reynolds numbers, as I do in figure 3 In this plot, an engineer needs to only compute one number (the Weber number) rather than two (the Ohnesorge and Reynolds numbers) to determine which regime a jet is in. A similar plot has been made by Faeth (1991, fig. 2) previously.

Some studies in table 1 focused on a single regime boundary rather than a complete diagram (Grant and Middleman, 1966, Kusui, 1969, Malot and Dumouchel, 2001, Miesse, 1955, and Sterling and Sleicher, 1975). The majority of previous studies classified regimes based on qualitative evaluation of images ( 9 out of 13 considered). The majority of previous studies also only considered a relatively small amount of data, e.g., 8 out of 13 studies considered had less than 100 data points in total, making their regime diagrams based on rather sparse data. For that reason, as much quantitative data as possible was compiled to construct the new regime diagram in this work. As shown in table 1, this work uses roughly an order of magnitude more data than any previous study. This gives the new regime diagram much more resolution and also range than previous regime diagrams. The Reynolds number ranges from $1.5 \times 10^{1}$ to $7.3 \times 10^{5}$ in this work, and the Weber number ranges from $2.0 \times 10^{0}$ to $4.1 \times 10^{5}$.

Rough pipe data from Kusui (1969) was used to determine the variation of the atomization regime boundary with the turbulence intensity. The turbulence intensity varied appreciably in

only a few additional previous studies. The estimation of the turbulence intensity will be discussed later in $\S 4.1$. In this study the turbulence intensity ranges from $4.9 \%$ to $12.7 \%$.

\section{ISSUES WITH PREVIOUS REGIME DIAGRAMS}

Figures 2 and 3 have many issues, the most significant of which will be detailed in this section. 


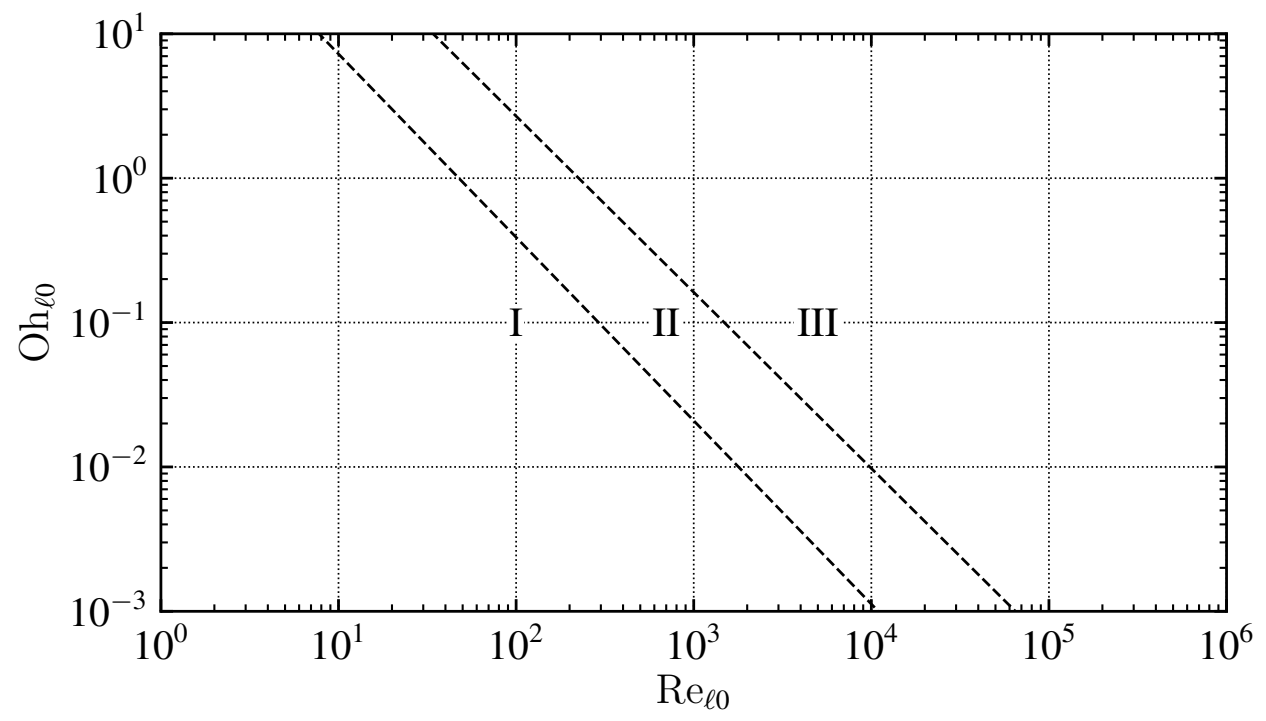

FIG. 2: A reproduction of the regime diagram of von Ohnesorge (2019), using Roman numerals for the regime names.

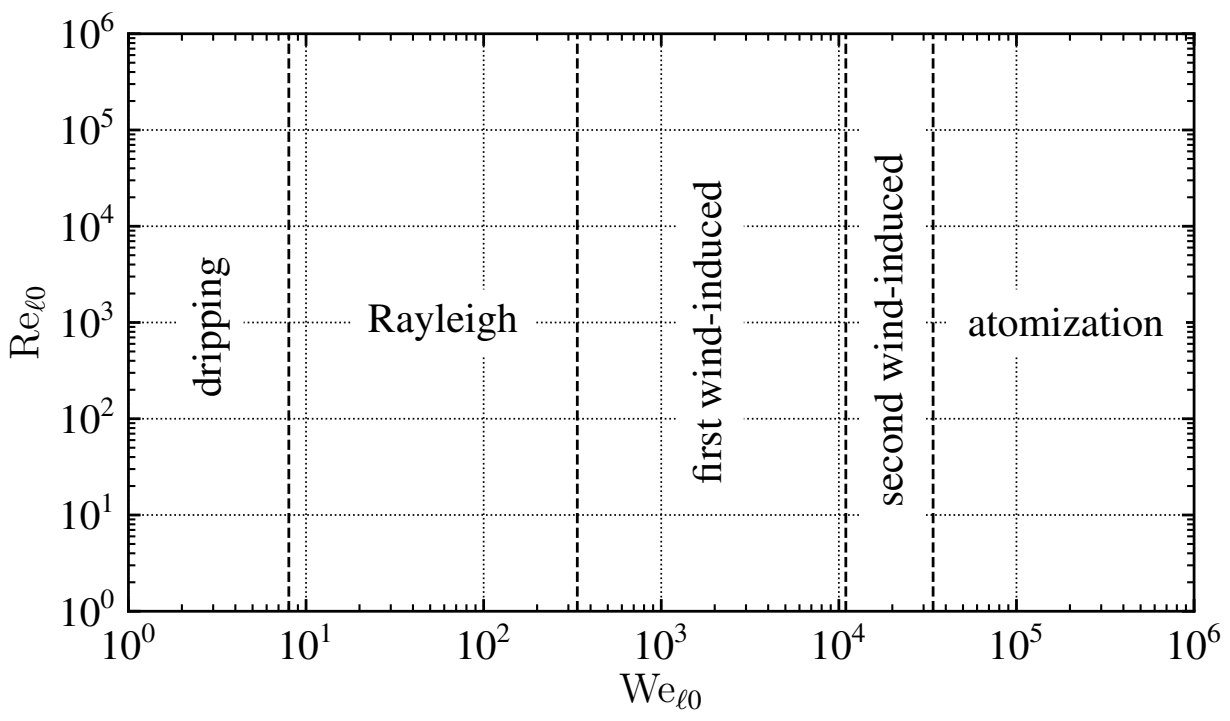

FIG. 3: The most popular regime diagram in liquid Weber number and liquid Reynolds number coordinates for liquid water injected into atmospheric air at $25^{\circ} \mathrm{C}$. These coordinates are preferred over those used in figure 2 because they simplify the regime diagram. Note that in this regime diagram, 3 of 4 of the regime boundaries will move if the liquid-gas density ratio is changed.

Volume 30, Issue $x, 2020$ 
Trettel

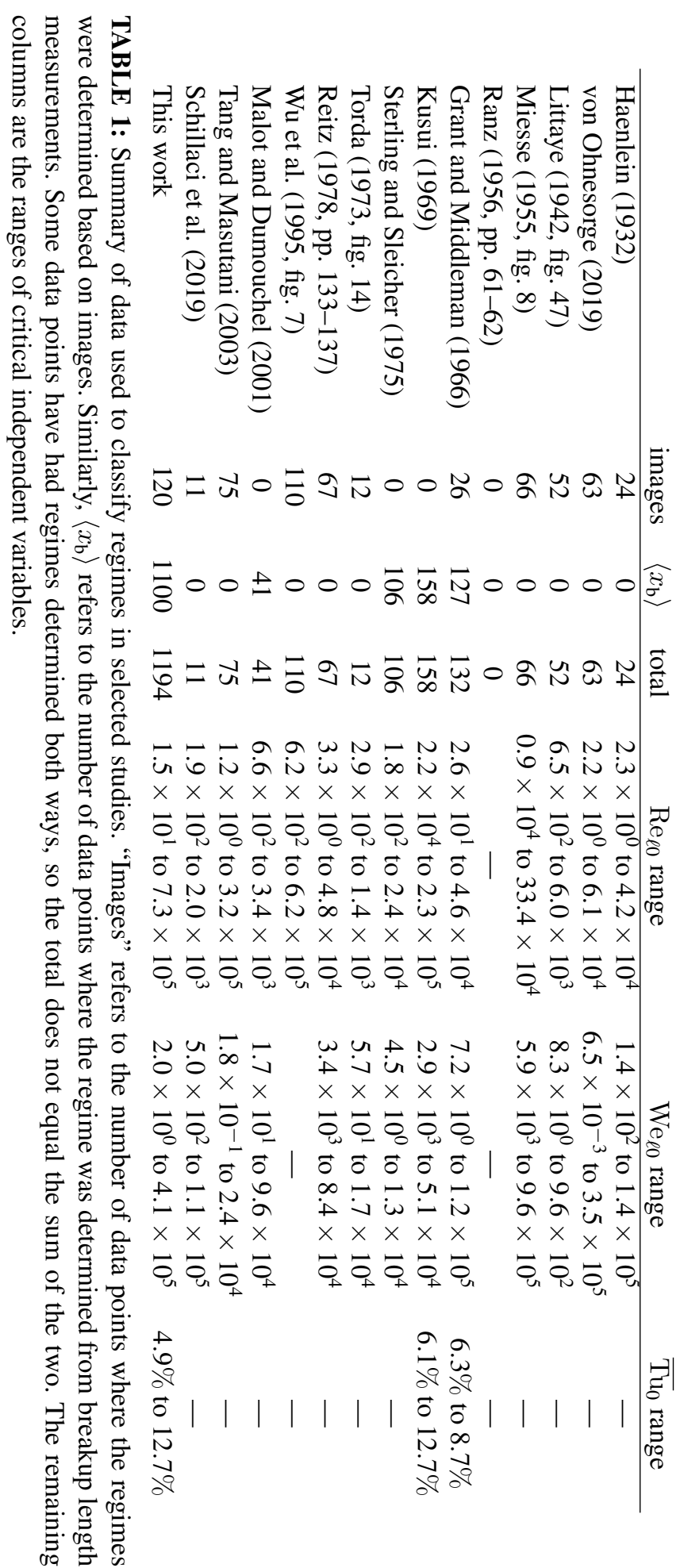




\subsection{Little data justifying most of the boundaries}

The most egregious problem with the most commonly used boundaries as represented in figure 3 is how little data they are based on. 3 out of 4 of the regime boundaries in figure 3 come from Ranz (1956, p. 61):

$$
\begin{aligned}
\text { dripping if } & \mathrm{We}_{\ell 0}<8 \\
\text { Rayleigh if } & \mathrm{We}_{\mathrm{g} 0}<0.4 \quad \text { and } \mathrm{We}_{\ell 0}>8 \\
\text { first wind-induced if } & 0.4<\mathrm{We}_{\mathrm{g} 0}<13 \\
\text { second wind-induced if } & 13<\mathrm{We}_{\mathrm{g} 0} .
\end{aligned}
$$

These boundaries are purely theoretical. There is no comparison of the boundaries against experimental data in Ranz's report - see table 1 . The derivations are missing from $\mid \mathrm{Ranz}$ 's report, but they appear to be based on simple scaling arguments using assumed physical mechanisms. Some of these assumptions end up being poor. For example, the assumption that the transition to the second wind-induced regime is caused by the influence of ambient gas is found to be false in $\S 3.3$ and $\S 4.10$ of this work. (Note that Ranz calls the second wind-induced regime atomization - a distinction between the two regimes was not made until later.) While the accuracy of these boundaries could be worse, they are appreciably less accurate than the new boundaries developed in this work.

\subsection{The most common atomization regime boundary was miscalculated}

The most popular atomization regime boundary criteria is $\mathrm{We}_{\mathrm{g} 0 \text {, crit }}=40.3$, and this boundary is used in figure 3. This boundary was developed from a functional form proposed by Littaye (1944) that was fitted by Miesse (1955, p. 1697L) to regime data classified visually. The criteria was not written as $\mathrm{We}_{\mathrm{g} 0 \text {, crit }}=40.3$ until the work of Reitz (1978, p. 8). As noted by Etzold (2019, pp. 77-78), We 0 ,crit $=40.3$ is inconsistent with Miesse's original criteria, which can be written as $\sqrt{\mathrm{We}_{\mathrm{g} 0 \text {,crit }} / 2}=6.35$, or, $\mathrm{We}_{\mathrm{g} 0 \text {, crit }}=80.6$. Reitz gives no derivation, but presumably the division by 2 was neglected.

Chigier and Reitz (1996, p. 113) imply that this criteria was derived in a different way using an alternative regime boundary equation from Miesse (1955, p. 1698L):

$$
\mathrm{Oh}_{10}=100 \mathrm{Re}_{\ell 0}^{-0.92} \text {. }
$$

Chigier and Reitz (1996) and Reitz (1978) claim this equation is consistent with $\mathrm{We}_{\mathrm{g} 0 \text {, crit }}=40.3$ because if $\mathrm{We}$ g0,crit is assumed to be constant then $\mathrm{We}$ g0,crit $\equiv \rho_{\ell} \bar{U}_{0}^{2} d_{0} / \sigma$ can be rewritten as

$$
\mathrm{Oh}_{10}=\left(\mathrm{We} \mathrm{g}_{\mathrm{g}, \mathrm{crit}} \frac{\rho_{\ell}}{\rho_{\mathrm{g}}}\right)^{1 / 2} \operatorname{Re}_{\ell 0}^{-1}
$$

so presumably $100 \approx \sqrt{\mathrm{We} e_{0, \text { crit }} \rho_{\ell} / \rho_{\mathrm{g}}}$. However, this implies a density ratio of $\rho_{\ell} / \rho_{\mathrm{g}} \approx 248$, which is inconsistent with Miesse's experiment. Miesse used water in ambient air and liquid nitrogen in air (Miesse, 1955, p. 1694L). Based on the numbers included in Miesse's paper, the density ratio for water in air was $\rho_{\ell} / \rho_{\mathrm{g}}=742$ and the density ratio for liquid nitrogen in air was $\rho_{\ell} / \rho_{\mathrm{g}}=671$. (Using instead $\mathrm{We} \mathrm{e}_{\mathrm{g} \text {, crit }}=80.6$ is even less accurate, implying that $\rho_{\ell} / \rho_{\mathrm{g}} \approx 124$.) Using the density ratio for water in air returns $\mathrm{We}_{\mathrm{g} 0 \text {, crit }}=12$. This is more consistent with $\operatorname{Ranz}$

Volume 30, Issue $x, 2020$ 
TABLE 2: Regime name conversions.

\begin{tabular}{|c|c|}
\hline New regime name & Old regime name \\
\hline dripping & dripping \\
laminar Rayleigh & Rayleigh \\
downstream transition & first wind-induced \\
turbulent Rayleigh & - \\
turbulent surface breakup & second wind-induced \\
(turbulent) atomization & atomization \\
\hline
\end{tabular}

(1956. p. 61), who proposes that $\mathrm{We}_{\mathrm{g} 0 \text {, crit }}=13$ as the onset of atomization, although as stated previously, that limit was later associated with the onset of the second wind-induced regime.

In summary, the existing regime boundaries are either based on no data at all, or based on a miscalculation. This situation is unacceptable, and the heavily data-driven regime diagram developed here is intended to remedy this.

\subsection{Regime names}

Table 2 shows the conversion between the regime names proposed in this work and the conventional regime names. While three are similar to the earlier names, in this work I use the phrase "turbulent surface breakup" instead of "second wind-induced", and the phrase "downstream transition" instead of "first wind-induced". The "wind-induced" regime names do not accurately describe the physical mechanisms involved. Many past researchers believe that breakup in these regimes is caused mainly by ambient gas effects (Dumouchel, 2008; Lin and Reitz, 1998, and Reitz, 1978), but my own analyses cast doubt on these assertions. To be more specific, in the "second wind-induced" (i.e., turbulent surface breakup) regime I find negligible influence of the ambient gas (see $\$ 4.10$ on the breakup length for the numbers), which makes the "windinduced" name seem inappropriate. Breakup in this regime appears to be caused by the internal turbulence of the jet. Sallam (2002, p. 94) agrees with this assessment. The so-called "first windinduced" regime appears to at least sometimes have a dependence on the ambient gas, though other factors like turbulence transition and velocity profile relaxation (both possibly influenced by the ambient environment) appear to be factors as well. However, without the second windinduced regime, a regime called the first wind-induced regime seems misplaced, so I've chosen the name "downstream transition" instead. This name is tentative as I believe there are multiple regimes contained within the downstream transition regime; see $\S 4.6$ for details.

\subsection{Hydrodynamic regime at nozzle outlet}

It is important to consider how the hydrodynamic regime (i.e., turbulent vs. laminar) of the flow at the nozzle outlet affects breakup regimes. One would expect turbulence transition to cause a change in the breakup regime. I first realized this after seeing that there was no turbulence transition in the nozzle in the original Ohnesorge diagram, figure 2 Based on the data I've compiled, which is limited to jets produced by long pipes, the transition from the downstream transition regime to the turbulent surface breakup regime appears to be caused by turbulence transition in the nozzle. This is most readily seen in the data of Grant and Middleman (1966, fig. 11, p. 675R), who classified the nozzle outlet flow as laminar or turbulent. Unfortunately, the most popular regime diagram as expressed through figure 3 does not distinguish between jets 
which are turbulent or laminar at the nozzle outlet, despite the clear effects the hydrodynamic regime has on the jet breakup.

In this work the new regime diagram has a critical Reynolds number for the start of transition inside the nozzle, $R_{\ell 0, \text { trans }}$, and also a second critical Reynolds number for the establishment of fully turbulent flow inside the nozzle, $R_{\ell 0}$,turb. The numbers used in figures 4 and 5 are 2300 and 4000, respectively. These will vary from system to system in difficult to predict ways, even for fully developed pipe flows (Mullin, 2011). For simplicity, it is convenient to use a single nozzle critical Reynolds number, $\mathrm{Re}_{\ell 0, \text { turb. }}$. Because the nozzle critical Reynolds number can vary greatly, breakup regime diagrams must consider the nozzle critical Reynolds number as a variable. Reviewing the literature on the nozzle critical Reynolds number of nozzles and pipes is prudent. A compilation of values observed in the literature is given in table 3 and these values will be discussed. Laminar flows at the outlet of a converging nozzle have been observed at Reynolds numbers two orders of magnitude higher that those seen in pipe flows. For example, Hoyt and Taylor (1977, fig. 2) observed laminar flow at the outlet of a converging nozzle at $\operatorname{Re}_{\ell 0}=2 \times 10^{5}$. With that being said, the critical Reynolds number for a nozzle flow can be comparable to that of a pipe flow. For example, appreciable turbulence intensities were measured by Lebedev (2019) for a nozzle presumably like a diesel injector at Reynolds numbers of about $1 \times 10^{3}$. One can assume the nozzle was rough ${ }^{\dagger}$ as Tonkonogiy et al. (1990) shows that the critical Reynolds number of a rough pipe can decrease below that of a smooth pipe:

$$
\operatorname{Re}_{\ell 0, \text { turb }}=\min \left[38\left(\frac{\epsilon}{d_{0}}\right)^{-0.8}, \sim 2000\right],
$$

where $\epsilon$ is the height of the roughness elements used in Tonkonogiy et al.'s experiments.

The nozzle critical Reynolds number is also a function of the nozzle length. van de Sande and Smith (1976 pp. 220R-221L, eqn. 10) conducted experiments and constructed the following empirical regression for the nozzle critical Reynolds number as a function of the dimensionless nozzle length $L_{0} / d_{0}$, valid for $1 \leq L_{0} / d_{0} \leq 100$ :

$$
\operatorname{Re}_{\ell 0, \text { turb }}=1.2 \times 10^{4}\left(\frac{L_{0}}{d_{0}}\right)^{-0.3} .
$$

The contraction ratio of these experiments was very large $\left(d_{\text {in }} / d_{0}>150\right)$, so presumably the flow was stabilized due to relaminarization or turbulence reduction in the contraction (Batchelor and Proudman, 1954 and Narasimha and Sreenivasan, 1979). With smaller contraction ratios, likely the nozzle critical Reynolds number is lower than implied by equation 8 . Further, because equation 8 is a power law, the calculated nozzle critical Reynolds number will not saturate at large $L_{0} / d_{0}$ as one might expect when the flow becomes fully developed. Consequently, to predict the nozzle critical Reynolds number if the nozzle length is longer than $100 d_{0}$ it is recommended to use $L_{0} / d_{0}=100$ instead of the actual nozzle length.

As a check on this equation, a regime plot of Wu et al.(1995, fig. 7) can be used to develop a very approximate nozzle critical Reynolds number equation $\left(\operatorname{Re}_{\ell 0 \text {,turb }} \approx 4.7 \times 10^{4}\left(L_{0} / d_{0}\right)^{-0.22}\right)$ which is similar to equation 8 . Equation 8 is recommended over this latter equation due to the likely better accuracy. One difference is worth noting: Wu et al. do not observe turbulence at the

\footnotetext{
${ }^{\dagger}$ Another possibility is that laminar flows could have appreciable turbulence intensities. Despite the name, the "turbulence intensity" is defined for any unsteady flow, not just turbulent flows. However, it seems unlikely that the turbulence intensity would be as high as it is in Lebedev s study.
}

Volume 30, Issue x, 2020 
nozzle outlet for $L_{0} / d_{0}<6$, but van de Sande and Smith do. The reason for this difference is unknown, and highlights the difficulty of predicting turbulence transition.

Interestingly, apparently all data in the classic regime study of von Ohnesorge (2019) was laminar at the nozzle outlet. This actually has major consequences for the regime diagram, which are discussed in $\$ 4.6$. It is possible that previous regime diagrams did not distinguish between laminar and turbulent flows because all flows observed were laminar at the nozzle outlet. This is not to say that initially laminar jets will never breakup up due to turbulence. These jets tend to transition to turbulence externally; they are merely initially laminar.

Some of the jet breakup data compiled in this work apparently had unusually high nozzle critical Reynolds numbers for pipe flows, i.e., for Eisenklam and Hooper (1958) and Sterling and Sleicher (1975), $\operatorname{Re}_{\ell 0 \text {,turb }}=\mathcal{O}\left(10^{4}\right)$. These studies were neglected for that reason, as they are inconsistent with other studies. It is not unheard of to have critical Reynolds numbers higher than those typical for pipe flows. Pfenninger (1961) was able to increase $\operatorname{Re}_{\ell 0 \text {,turb }}$ to $\mathcal{O}\left(10^{5}\right)$ for a pipe by taking care to eliminate flow disturbances. Mullin (2011) notes that a high critical Reynolds number is a good measure of the quality of an experimental facility.

\subsection{Turbulence intensity effects}

Reitz (1978, p. 9) notes that the conventional regime boundaries do not correctly predict the regime in water jet cutting, where the jets appear to be considerably more stable and consequently in "earlier" regimes than the standard criteria would suggest. Reitz(1978, p. 9) attributes these violations to "nozzle geometry effects". The turbulence intensity can explain this discrepancy, as the turbulence intensity would be lower in cutting water jets than the fuel sprays typically studied. The more recent study of Tafreshi and Pourdeyhimi (2003) shows another violation of the earlier regime boundaries likely due to turbulence intensity effects.

Existing regime diagrams suffer from poor reproducibility due to neglecting several turbulence related variables like the nozzle critical Reynolds number, jet critical Reynolds number, and turbulence intensity. General regime criteria need to take into account fundamental physics, and not be mere regressions which apply only to particular cases. Consequently, a goal of this work is to include the turbulence intensity as a variable wherever possible.

\subsection{Qualitative vs. quantitative classification}

As previously mentioned, regimes are typically defined in qualitative ways based on images or vague descriptions (e.g., see table 4). This is particularly problematic for the atomization regime, as it's superficially similar to the turbulent surface breakup regime, just more vigorous in some sense. Consequently, I use quantitative criteria to determine the regimes based on breakup length in table 5 (discussed earlier as well). In this work I also unify the visual and breakup length regimes so that each "breakup length" regime has an associated appearance. However, it may not be always possible to go the other direction, from the appearance to the regime, due to ambiguities in the appearance. Note that the choice of the breakup length as the quantity of interest does not imply that the breakup length is superior to other quantities for the purposes of classifying regimes - the breakup length merely is ubiquitous in the literature.

The next problem is partly caused by the superficiality of qualitative regime determination. 


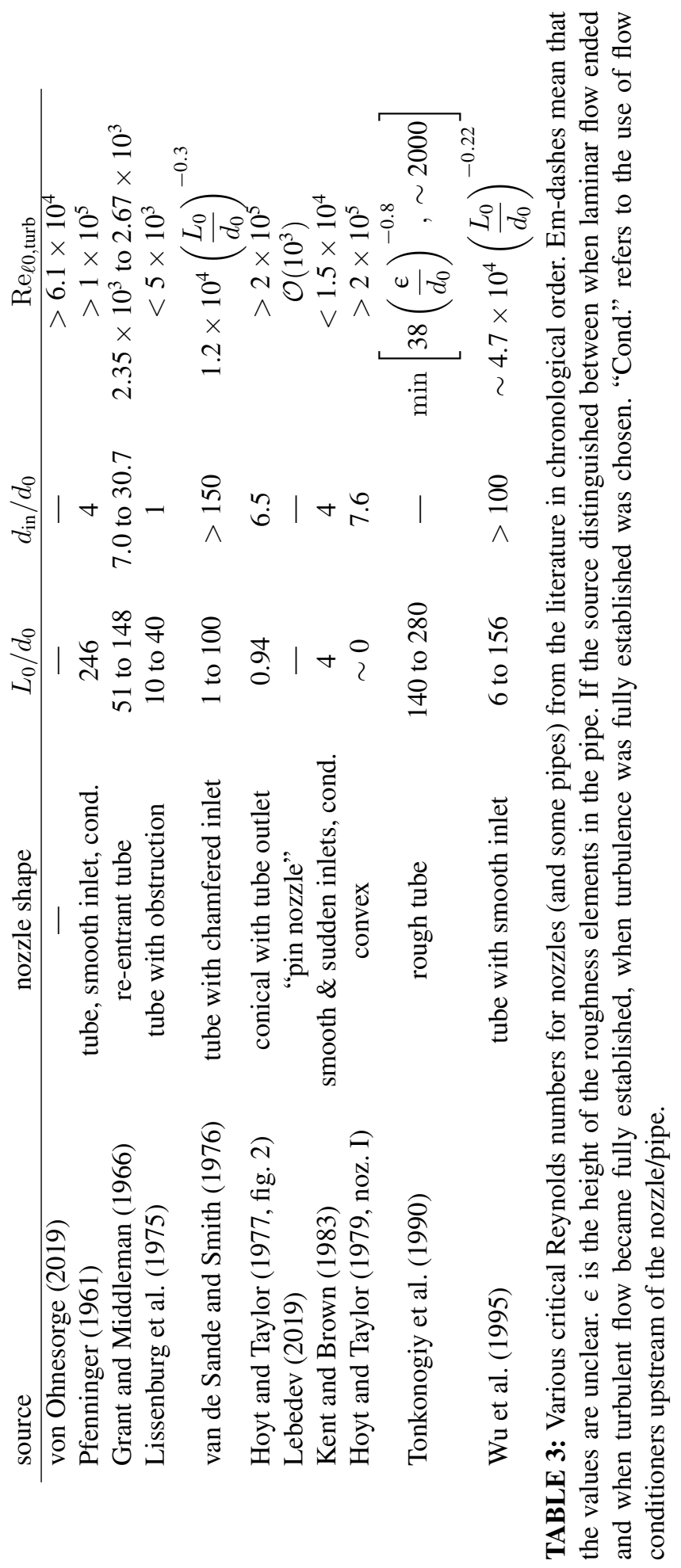

Volume 30, Issue x, 2020 
TABLE 4: Qualitative characteristics of each regime.

\begin{tabular}{|c|c|}
\hline Regime name & Appearance \\
\hline dripping & slow formation of droplets at the nozzle outlet \\
laminar Rayleigh & symmetric breakup into large droplets \\
downstream transition & varies from Rayleigh-like to abrupt breakup \\
turbulent Rayleigh & similar to laminar Rayleigh, but turbulent \\
turbulent surface breakup & small surface disturbances causing breakup \\
(turbulent) atomization & larger spray angle than turbulent surface breakup \\
\hline
\end{tabular}

TABLE 5: Quantitative characteristics of each regime.

\begin{tabular}{|c|c|c|}
\hline Regime name & $\left\langle\boldsymbol{x}_{\mathrm{b}}\right\rangle / \boldsymbol{d}_{0}$ & $\boldsymbol{D}_{32}$ \\
\hline dripping & $\propto\left(\frac{\mathrm{Fr}_{0}}{\mathrm{We}_{\ell 0}}\right)^{1 / 3}$ & $\mathcal{O}\left(d_{0}\right)$ \\
laminar Rayleigh & $\propto \mathrm{We}_{\ell 0}^{1 / 2}+3 \frac{\mathrm{We}_{\ell 0}}{\mathrm{Re}_{\ell 0}}$ & $\mathcal{O}\left(d_{0}\right)$ \\
downstream transition & decreasing with $\bar{U}_{0}$ & varies \\
turbulent Rayleigh & $\propto \operatorname{arccsch}\left(C_{v} \overline{\mathrm{Tu}}_{0} \mathrm{We}_{\ell 0}^{1 / 2}\right) \mathrm{We}_{\ell 0}^{1 / 2}$ & $\mathcal{O}\left(d_{0}\right)$ \\
turbulent surface breakup & $\propto \mathrm{We}_{\ell 0}^{1 / 3}$ & $<d_{0}$ \\
(turbulent) atomization & $\propto\left(\frac{\rho_{\ell}}{\rho_{\mathrm{g}}}\right)^{C_{\rho}}$ & $\ll d_{0}$ \\
\hline
\end{tabular}

\subsection{A missing regime and the varying regime progression}

When a liquid jet is in the Rayleigh regime and the velocity is increased (all else equal), convention stipulates that the jet will eventually enter the downstream transition regime (again, previously called the first wind-induced regime). This is not necessarily true. The "regime progression" - that is, how the regimes change as the jet velocity increases - is not universal.

While the majority of jets previously studied will enter the downstream transition regime after "the" Rayleigh regime, some researchers have identified a turbulent Rayleigh regime that is different from the conventional laminar Rayleigh regime (see figure 6) and can follow the laminar Rayleigh regime instead of the downstream transition regime (Asset and Bales. 1951; Lafrance et al., 1974; Mansour and Chigier, 1994a; Phinney, 1973, 1975, Sallam et al., 2002, Sterling and Abbott, 1981, van de Sande and Smith 1976). This regime is missing from the conventional Ohnesorge diagram. The turbulent Rayleigh regime is sometimes superficially visually similar to the downstream transition regime. The downstream transition regime takes various forms - see figures 7 through 9 Compare figure 7, a jet early in the downstream transition regime, against figure 10 a jet in the turbulent Rayleigh regime. The two regimes appear to be visually similar to the laminar Rayleigh regime, although less regular.

However, the breakup length decreases as velocity increases in the downstream transition regime, but increases as velocity increases in the turbulent Rayleigh regime. Typically, the first wind-induced regime is defined as having a decreasing breakup length, e.g., by Reitz (1978, fig. 1.1) and Dumouchel (2008, fig. 1). Many studies have examined the first peak in the breakup length curve, motivated by the downstream transition regime; see Dumouchel (2008, pp. 376379) for a review of these studies. However, the breakup length can also peak due to turbulence 
transition in the nozzle ( $\left.\operatorname{Re}_{\ell 0}>\operatorname{Re}_{\ell 0 \text {,trans }}\right)$. And once turbulent flow is established, the breakup length then will increase roughly proportional to $\mathrm{We}_{\ell 0}^{1 / 2}+3 \mathrm{We}_{\ell 0} / \mathrm{Re}_{\ell 0}$, just like in the laminar Rayleigh regime, albeit with a lower constant of proportionality - see $\$ 4.8$. As such, I define the downstream transition regime as laminar at the nozzle outlet, in contrast to previous researchers like Reitz (1978, p. 24). The data compilation detailed in this work shows that the downstream transition regime only appears when the flow is laminar at the nozzle outlet.

Conflating the downstream transition and turbulent Rayleigh regimes is not uncommon. For example, Reitz (1978, pp. 24-25) suggests that the experiments of Phinney (1973) are in the downstream transition regime, but these experiments are actually largely in the turbulent Rayleigh regime. Similarly, Dumouchel (2008, p. 378) does not consider the turbulent Rayleigh regime as a possibility, however, the downstream transition boundary Dumouchel uses in effect differentiates between the turbulent Rayleigh and downstream transition regimes.

The existence of the turbulent Rayleigh regime is just one example of how the regime progression is not universal. Examine the three diagonal lines in figure 5. The middle line is the "conventional" case, that is, where the jet transitions from the (laminar) Rayleigh regime to the downstream transition regime, then to the turbulent surface breakup regime (shown in figure 11), and then to the atomization regime. The trends in the breakup length for this case are shown in figure 12 The "conventional" case is only one of several possibilities. A larger pipe nozzle with a low viscosity and high surface tension liquid (i.e., water) follows the path shown by the upper diagonal line in figure 5. This case never enters the downstream transition regime, as seen in figure 13. A more typical case is that seen for a lower surface tension liquid like gasoline with a smaller nozzle, corresponding to the lower diagonal line. This case, which is typical for fuel sprays, never enters the turbulent surface breakup regime, as seen in figure 14

Another way in which the regime progression is not universal comes through the influence of the nozzle critical Reynolds number. This has been recognized by previous researchers. Eisenklam and Hooper (1958, fig. 14) noticed that if they add a turbulence trip inside of their nozzle, they can avoid what they called "bursting breakup", which is a particularly violent form of breakup in the downstream transition regime (see figure 8 for an example). Similarly, Hoyt and Taylor (1985) suggest moving turbulence transition inside of the nozzle, or in other words, decreasing the nozzle critical Reynolds number, to avoid very vigorous breakup (identical to "bursting breakup") apparently caused by external turbulence transition.

\subsection{Breakup onset location in atomization}

The atomization regime is sometimes defined as when the breakup onset location is very small, indistinguishable from zero (Reitz and Bracco, 1986, p. 235). This definition is unsatisfactory as the breakup onset location can also be small in the turbulent surface breakup regime. The breakup onset location theory in Trettel (2020a) applies to both the turbulent surface breakup and atomization regimes. Consequently, I define the atomization regime based on more obvious quantitative criteria: the breakup length curve. I define the turbulent surface breakup regime as having an increasing power law behavior in the breakup length as a function of $\mathrm{We}_{\ell 0}$ (i.e., $\left\langle x_{\mathrm{b}}\right\rangle / d_{0} \stackrel{\sim}{\sim} \mathrm{We}_{\ell 0}^{1 / 3}$ ), and the atomization regime is defined as a plateau as $\mathrm{We}_{\ell 0}$ increases, with all else constant and neglecting cavitation and compressibility effects (to be discussed in $\$ 4.11$.

Volume 30, Issue $x, 2020$ 


\subsection{Problems with other regime diagrams}

$\mathrm{Wu}$ and Faeth (1993) suggests that $\rho_{\ell} / \rho_{\mathrm{g}}<500$ is a reasonable criteria for the onset of aerodynamic effects, in this work called the atomization regime. Magnotti (2017) later suggested that $\rho_{\ell} / \rho_{\mathrm{g}}<300$ is a more accurate criteria for the onset of aerodynamic effects. Purely density ratio based criteria are not accurate, as increasing the bulk velocity alone can change the regime from turbulent surface breakup to atomization (Kusui, 1969 and Sallam et al., 2002). The differences in the boundaries observed by Wu and Faeth and Magnotti likely could be attributed to differences in the Weber numbers and turbulence intensities.

Reitz developed a criteria for the onset of the atomization regime which is claimed to consider the effects of nozzle geometry (Lin and Reitz, 1998, pp. 93-94). The criteria uses a regression for a model parameter based on Reitz's experiments. The only nozzle geometric parameter used was the nozzle length-to-diameter ratio, $L_{0} / d_{0}$. This neglects the large impact that the contraction ratio $d_{\text {in }} / d_{0}$ can have as discussed in $\$ 3.4$. Rather than using nozzle geometry, the model would generalize better if nozzle outlet turbulence intensity and nozzle critical Reynolds number were used (and be further improved by the inclusion of other variables like the velocity profile, etc.).

\section{PROPOSED REGIME DIAGRAM AND INFORMATION ON EACH REGIME}

Figure 4 is the proposed regime diagram. A sample of data for high liquid-gas density ratios and smooth pipe nozzles is shown in figure 5 to give a sense for how well these boundaries fit the data. In contrast with figure 4, which is for constant turbulence intensity, in figure 5 the turbulence intensity is a function of the Reynolds number, which is why some of the regime boundaries are not straight lines as they are in figure 4.

Individual regime boundaries were developed in various ways, using all data available for that boundary (i.e., not only high density ratio data as in figure 4), as described in the corresponding section for that boundary.

A warning: The proposed regime diagram is not meant to be used as presented to determine the regime. It is a schematic used to organize knowledge about the regimes. Regime determination is best done with the empirical equations developed in this work for each regime boundary. This is because the regime boundaries will move depending on the configuration. Figure 4 is only appropriate for pipe nozzles at high liquid-gas density ratios. Additionally, this regime diagram is not expected to be perfectly accurate, so engineers should check its prediction against the behavior of the jet (visual description and/or breakup length trend) to confirm the prediction.

\subsection{Data compilation}

The proposed regime diagram is based on a compilation of data available at GitHub (Trettel, 2020b). The GitHub repository will contain the latest data, regime diagrams, and regressions as this data compilation is updated in the future. The description and motivations of the data compilation are described in detail in Trettel (2019) and will only be briefly described here. This data compilation uses "pipe jet" data, where the nozzle is simply a long pipe. Pipe jets were chosen for their ubiquity in the literature, their high reproducibility (due to fully developed flows being a universal state), and because the turbulence intensity can be determined for pipes given the friction factor.

Data from 22 studies was compiled (Arai et al., 1985, Asset and Bales, 1951, Betchov and Clutter. 1955, Chen and Davis, 1964; Eisenklam and Hooper, 1958, Grant and Middleman. 


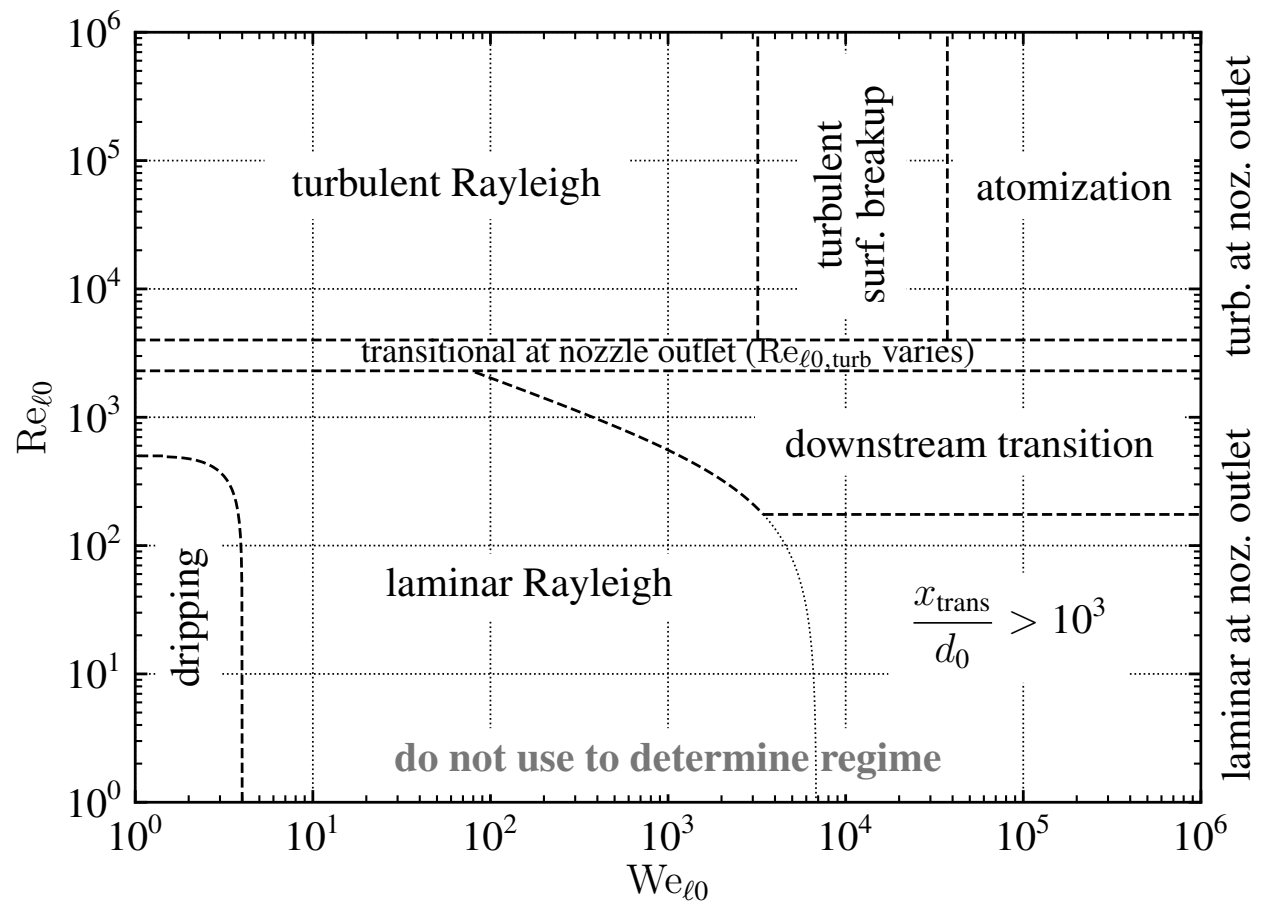

FIG. 4: Schematic regime diagram at low ambient densities for illustration purposes only. Do not use this plot to determine the regime. Regime boundaries are very approximate and apply only for a special case. More general regime boundary equations are given in the text. The nozzle critical Reynolds number will typically be an order of magnitude or more higher than in this plot, which is based on long pipe nozzles (fully developed pipe flow) that have atypically low critical Reynolds numbers - see table 3 . Constant high density ratio $\left(\rho_{\ell} / \rho_{\mathrm{g}}=1000 / 1.2\right)$ corresponding approximately to water-air at standard temperature and pressure. The dripping boundary is also for water-air systems. Turbulence intensity is $5 \%$. Turbulent regime boundaries should vary with Reynolds number in a smooth pipe due to variation of turbulence intensity with the Reynolds number — see figure 5 .

Volume 30, Issue x, 2020 

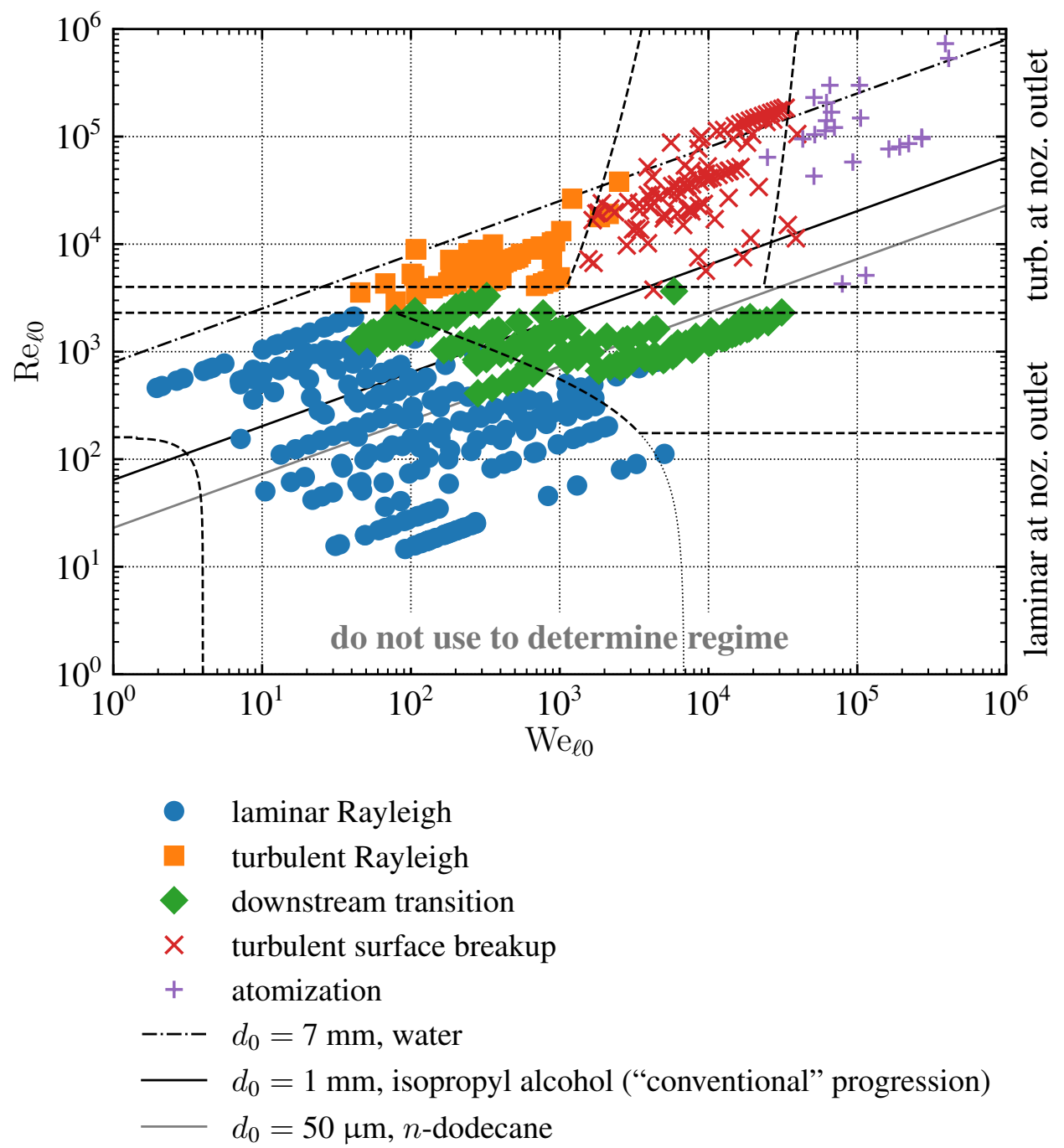

FIG. 5: Regime diagram for smooth pipe nozzles with data and example lines showing the regime progression for three different cases. Transitional regimes removed for clarity. Data from Eisenklam and Hooper (1958) and Sterling and Sleicher (1975) removed due to abnormally high nozzle critical Reynolds numbers. 564 data points. In contrast with figure 4 the turbulence intensity is now a function of the Reynolds number (as is the case for a smooth pipe flow), and consequently the turbulent regime boundaries vary with Reynolds number. High density ratio data only $\left(\rho_{\ell} / \rho_{\mathrm{g}}>500\right)$. Atomization regime boundary for $\rho_{\ell} / \rho_{\mathrm{g}}=1000 / 1.2$. Dripping regime boundary for $n$-dodecane. 


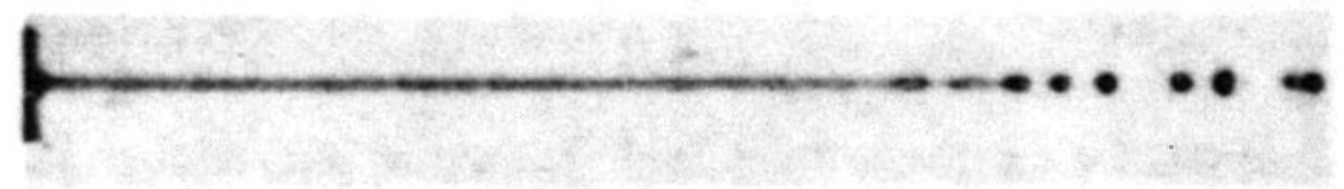

FIG. 6: Image of a water jet in the laminar Rayleigh regime from Asset and Bales (1951 fig. 1). Note the breakup is caused by disturbances symmetric to the jet axis. Flow is from left to right. (Public domain image.)

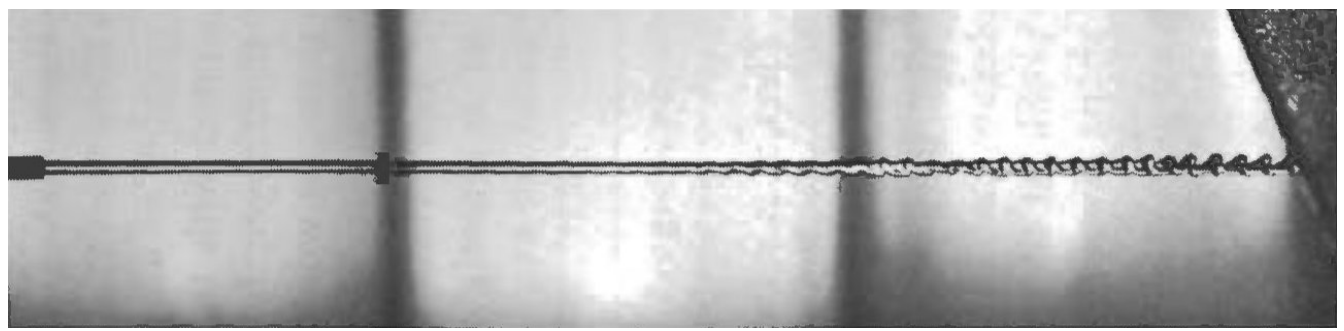

FIG. 7: Image of a water jet early in the downstream transition regime from $\operatorname{Rupe}(1962$ fig. 4c). Flow is from left to right. (Image used under license.)

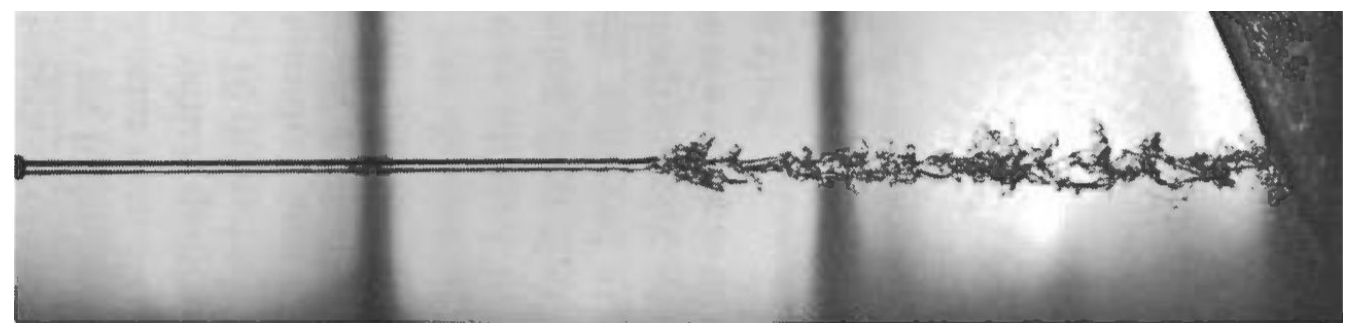

FIG. 8: Image of a water jet late in the downstream transition regime from Rupe (1962 fig. 4d) which is atomization-like. Note that the breakup is much more vigorous than that seen in figure 7 Flow is from left to right. (Image used under license.)

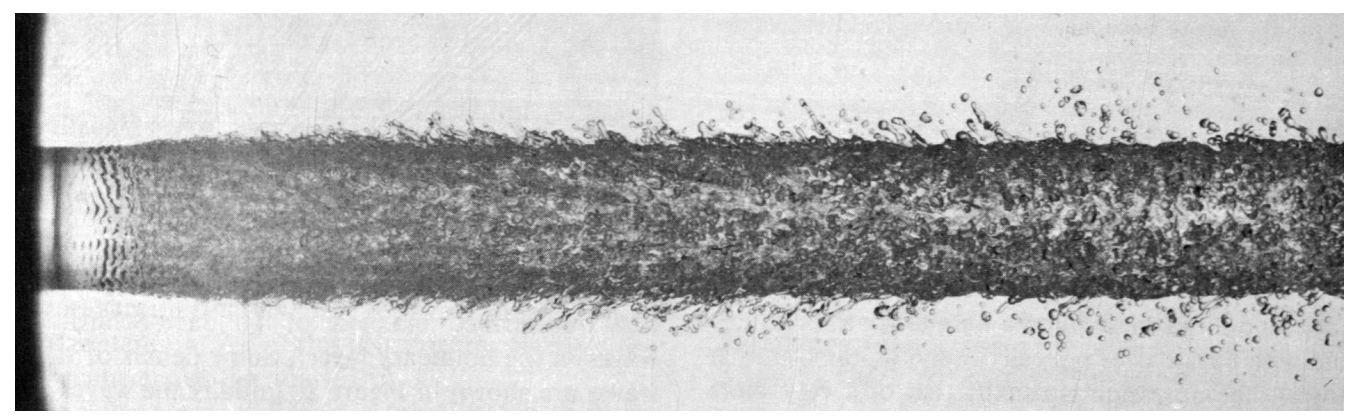

FIG. 9: Image of a water jet in the downstream transition regime from Hoyt and Taylor (1982 fig. 1). Flow is from left to right. Here the breakup is more vigorous than that seen in figure 7 but less vigorous than figure 8 This jet is in a regime similar to the turbulent surface breakup regime. Unlike the other images, which use pipe nozzles, this for a nozzle with a length-to-diameter ratio $\left(L_{0} / d_{0}\right)$ of 1 . (Public domain image.)

Volume 30, Issue x, 2020 


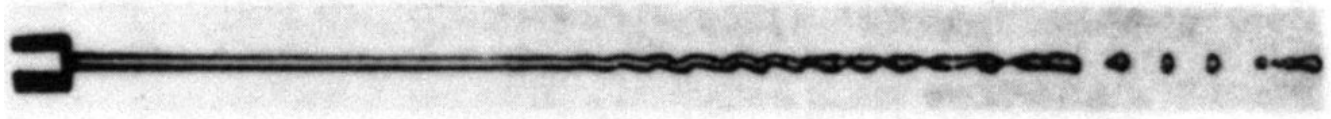

FIG. 10: Image of a water jet in the turbulent Rayleigh regime from Asset and Bales (1951, fig. 10). Flow is from left to right. (Public domain image.)

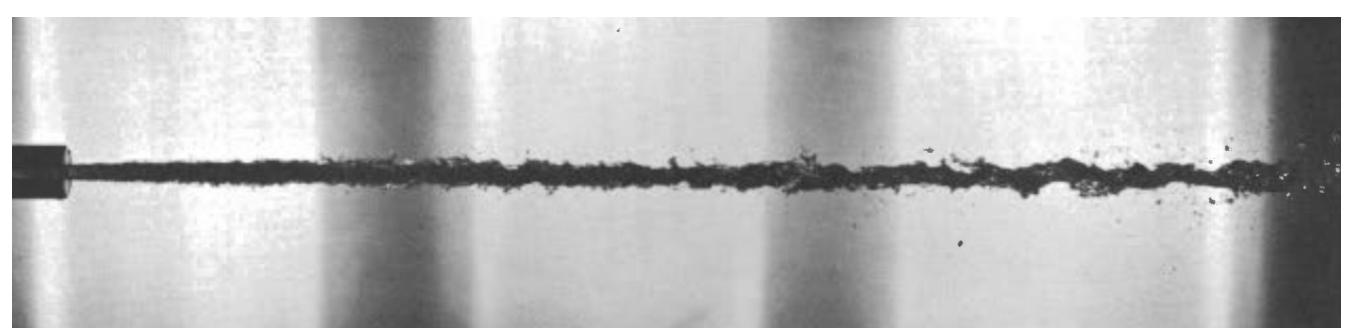

FIG. 11: Image of a water jet in the turbulent surface breakup regime from Rupe (1962, fig. 15c). Flow is from left to right. (Image used under license.)

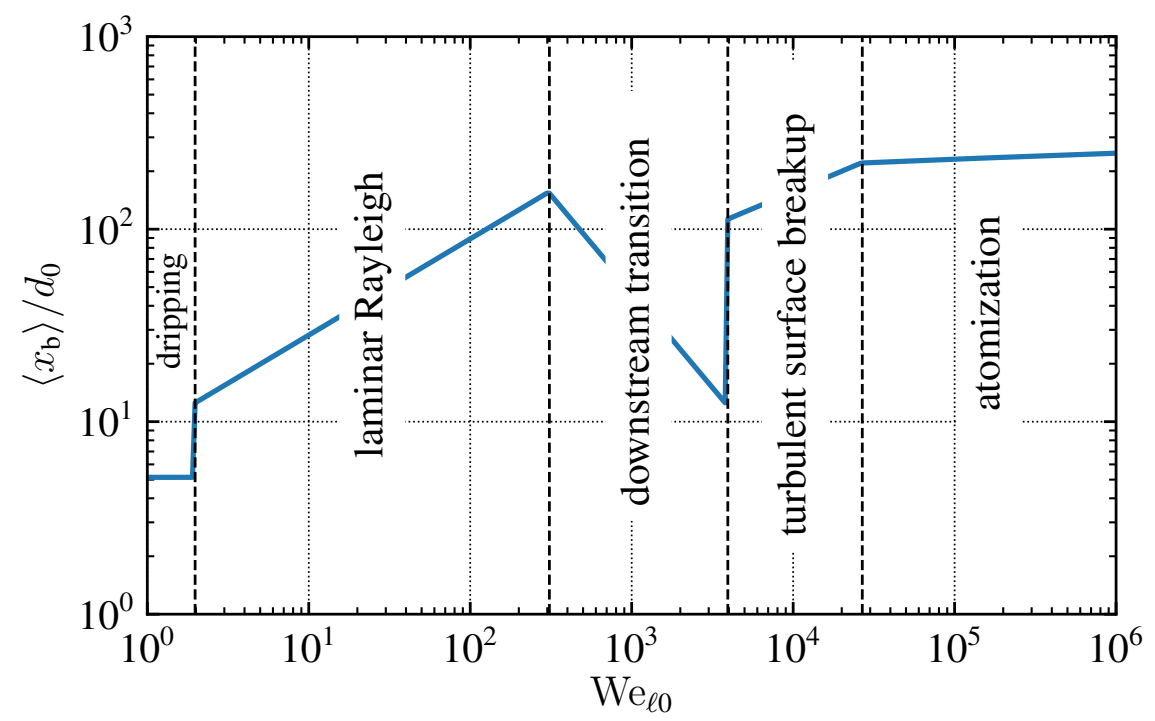

FIG. 12: Schematic "stability curve" for the "conventional" pipe nozzle case in figure 5 (center diagonal line). Pure isopropyl alcohol for pipe nozzle with $d_{0}=1 \mathrm{~mm}$. Regressions for the breakup length $\left\langle x_{\mathrm{b}}\right\rangle$ from the text are used in each marked regime above. Breakup lengths for the downstream transition regime are approximate, and the real stability curve will be smoother. Note that like in figure 5, the turbulence intensity $\overline{\mathrm{Tu}}_{0}$ and Reynolds number $\mathrm{Re}_{\ell 0}$ are changing as the Weber number $\mathrm{We}_{\ell 0}$ changes. 


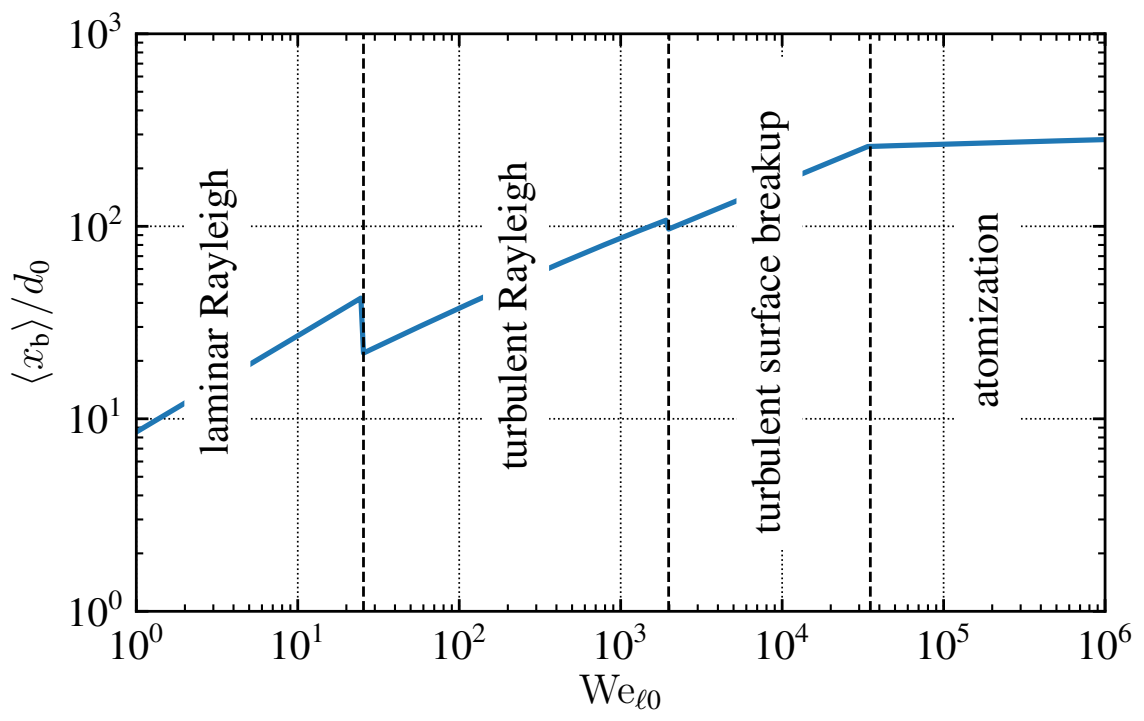

FIG. 13: Schematic "stability curve" for the pipe nozzle $d_{0}=6 \mathrm{~mm}$, water case in figure 5 (top diagonal line). See figure 12 for details.

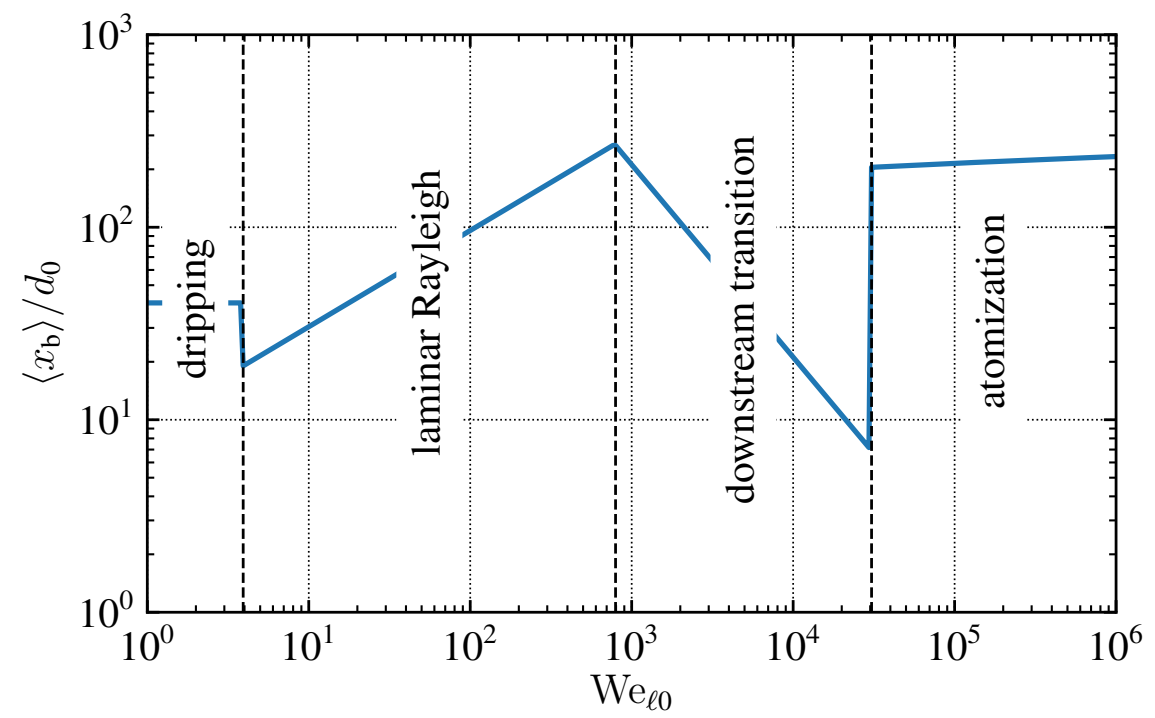

FIG. 14: Schematic "stability curve" for the pipe nozzle $d_{0}=50 \mu \mathrm{m}, n$-dodecane (similar to gasoline engine nozzle but with a lower $\mathrm{Re}_{\ell}$,turb) case in figure 5 (bottom diagonal line). In an actual engine nozzle, the same regime progression will be seen as the order is preserved if the nozzle critical Reynolds number is increased. See figure 12 for other details.

Volume 30, Issue $x, 2020$ 
1966; Hoyt and Taylor, 1980, 1985, Kim and Mills, 1989, Kusui, 1969, Mansour and Chigier, 1994a; Phinney, 1973, 1975; Phinney and Humphries 1970; Ruff 1990; Rupe, 1962, Sallam et al. 2002, Shimizu et al. 1984; Sterling and Sleicher 1975; Trettel, 2020c, Wu et al., 1983. 1992). To be clear, about $98.5 \%$ of the data in this compilation comes from the open literature and is not new to this work.

To limit the scope of the data compilation, only data where $\rho_{\ell} / \rho_{\mathrm{g}}>1$ was included in the data compilation. It is likely that the regime diagram is appreciably different at low density ratios - the turbulent surface breakup regime for instance might completely cease to exist as the critical Weber number for atomization decreases with the density ratio.

Turbulence intensity (defined in this work as $\overline{\mathrm{Tu}}_{0} \equiv\left(2 \bar{k}_{0} / 3\right)^{1 / 2} / \bar{U}_{0}$ ) was estimated using a regression between the friction factor and $\overline{\mathrm{Tu}}_{0}$ for fully developed pipe flows: $\overline{\mathrm{Tu}}_{0}=0.366 f^{0.459}$ ( 9 smooth and 8 rough points, $R^{2}=0.975$ ) (Trettel, 2018, p. 6). Generally the turbulence intensity is roughly constant for smooth pipe flows. Rough pipe data from Kusui (1969) was used to vary the turbulence intensity from $4.9 \%$ to $12.7 \%$. Note that Kusui had an 8.75 diameter smooth section after the rough section, which complicates the estimation of the turbulence intensity. The turbulence intensity was calculated as if this smooth section did not exist because that assumption best matched an alternative breakup length data source that included rough turbulence intensity estimates - see $\S 4.10$. Due to this uncertainty, new experiments with turbulence intensity variation are needed to supplant this data.

\subsection{Regimes and their physical mechanisms}

More detailed descriptions and analysis of each regime follows, going from left to right in figure 4 starting with the regimes with flows that are laminar at the nozzle outlet and following with the regimes with flows that are turbulent at the nozzle outlet.

When applicable, regressions for the breakup length and other quantities of interest will be presented. The reader is referred to Trettel (2019, 2020a) for details on the choice of the functional forms for the regressions.

\subsection{Dripping regime and transition to jetting}

Grant and Middleman (1966, fig. 1), McCarthy and Molloy (1974, fig. 2), Dumouchel (2008, fig. 1), and Lefebvre and McDonell (2017, fig. 2.13) suggest that the breakup length in the dripping regime increases considerably with velocity. This seems unlikely. While the breakup length has not been measured in the dripping regime to my knowledge, presumably it is roughly proportional to the droplet diameter. Assuming that the nozzle is very thin, the simple theory of Tate as described by Clanet and Lasheras (1999, p. 308) suggests that approximately

$$
D \propto\left(\frac{\sigma d_{0}}{\rho_{\ell} g}\right)^{1 / 3} .
$$

If $\left\langle x_{\mathrm{b}}\right\rangle \propto D$ in the dripping regime, then we might expect

$$
\frac{\left\langle x_{\mathrm{b}}\right\rangle}{d_{0}} \propto\left(\frac{\sigma}{\rho_{\ell} g d_{0}^{2}}\right)^{1 / 3}=\left(\frac{\mathrm{Fr}_{0}}{\mathrm{We}_{\ell 0}}\right)^{1 / 3},
$$

which does not vary with jet bulk velocity, $\bar{U}_{0}$. Possibly other effects will change this result, but likely the breakup length varies little with the jet bulk velocity in the dripping regime. 
The transition from dripping to "jetting" (laminar Rayleigh regime) has been studied extensively in the past. Clanet and Lasheras (1999) develop the following regime boundary for the end of dripping based on their theory and validate it with an extensive series of experiments:

$$
\mathrm{We}_{\ell 0, \text { crit }}=4 \frac{\mathrm{Bo}_{\text {outer }}}{\mathrm{Bo}}\left[1+K \mathrm{BoBo}_{\text {outer }}-\left(\left(1+K \mathrm{BoBo}_{\text {outer }}\right)^{2}-1\right)^{1 / 2}\right]^{2},
$$

where $\mathrm{Bo}^{2} \equiv \rho_{\ell} g d_{0}^{2} /(2 \sigma)$ and $\mathrm{Bo}_{\text {outer }}^{2} \equiv \rho_{\ell} g d_{\text {outer }}^{2} /(2 \sigma)$ define the Bond numbers for the inside and outside of the nozzle outlet ( $d_{\text {outer }}$ is the outer diameter), respectively, and $K$ is a constant which equals 0.37 for water injected into air.

Equation 11 is plotted in figures 4 and 5 for water and $n$-dodecane, respectively, with $d_{\text {outer }}=$ $d_{0}$. Note that while Clanet and Lasheras s theory is valid in the high Reynolds number limit only, it is possible to calculate a Bond number consistent with the Reynolds and Weber numbers, as was done in these plots.

\subsection{Laminar Rayleigh regime}

A jet in the laminar Rayleigh regime is shown in figure 6 . The theory of Weber (2019) accurately describes the breakup of jets in this regime. The equation for the breakup length in Weber's theory is

$$
\frac{\left\langle x_{\mathrm{b}}\right\rangle}{d_{0}}=C_{\mathrm{LR}}\left(\mathrm{We}_{\ell 0}^{1 / 2}+3 \frac{\mathrm{We}_{\ell 0}}{\mathrm{Re}_{\ell 0}}\right) .
$$

The term $C_{\mathrm{LR}}$ is an empirical coefficient that applies only for the laminar Rayleigh case. In the turbulent Rayleigh case the notation $C_{\mathrm{TR}}$ will be used.

Based on limited data from Haenlein (1932), Weber (2019 p. 24) recommends $C_{\mathrm{LR}}=12$ for the laminar Rayleigh regime. Grant and Middleman (1966, p. 673L) state that $C_{\mathrm{LR}}=13.4$ fits their data better. Grant and Middleman further suggest that $C_{\mathrm{LR}}$ is a function of $\mathrm{Oh}_{\ell 0}$. However, Kroesser and Middleman (1969, p. 385L) note that the variation in $C_{\mathrm{LR}}$ as a function of $\mathrm{Oh}_{\ell 0}$ is "so weak that it probably does not warrant detailed investigation". Along those lines, fitting data from multiple studies compiled in this work returns $C_{\mathrm{LR}}=8.51$ (247 points, $\left.R^{2}=0.942\right)$. A comparison of the available data to the regression is in figure 15 .

Weber's theory predicts that the size of the droplets in this regime is

$$
D=\left(\frac{3 \sqrt{2}}{2} \pi \sqrt{1+3 \mathrm{Oh}_{\ell 0}}\right)^{1 / 3} d_{0}
$$

and this equation is generally regarded as accurate when satellite droplets are negligible.

\subsection{Transition to the downstream transition regime}

The conventional explanation for why the laminar Rayleigh regime ends and the downstream transition regime begins is that aerodynamic forces become significant in the downstream transition regime, however, this is only one of several possibilities as will be explained. Early models of the aerodynamic effects variety were developed by Weber (2019) and Sterling and Sleicher (1975). A recent popular approach is by Dumouchel and co-workers, based around the idea that the transition to the downstream transition regime (and also the turbulent Rayleigh regime) can be found through the use of an ad hoc effective gas density, $\rho_{\mathrm{g}}^{*}$ (Dumouchel, 2008, p. 378). However, Malot and Dumouchel 2001, p. 231) admit that there is no theoretical justification for $\rho_{\mathrm{g}}^{*}$

Volume 30, Issue x, 2020 


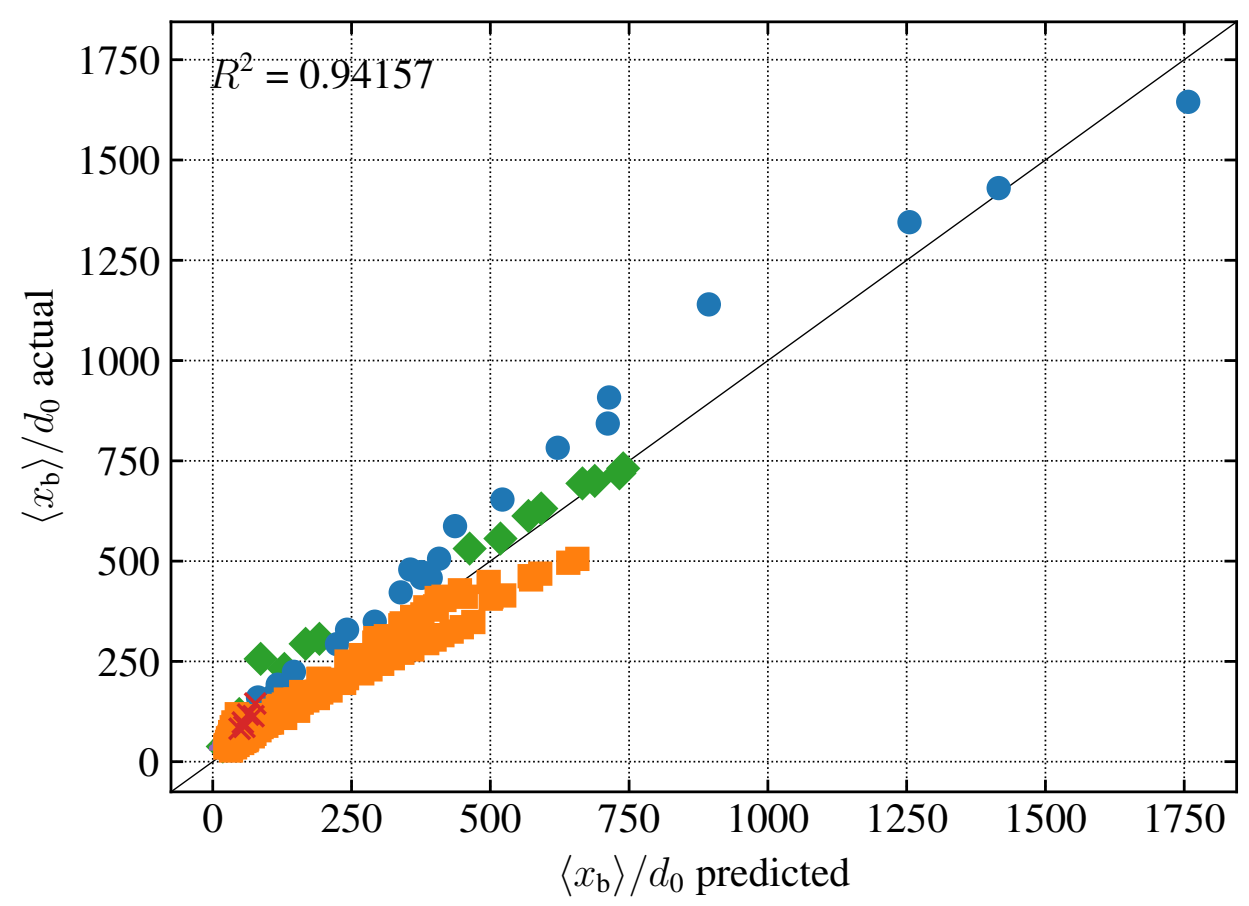

$\begin{array}{ll}+ & \text { Sterling and Sleicher (1975) (16 pts.) } \\ + & \text { Mansour and Chigier (1994a) (20 pts.) } \\ + & \text { Grant and Middleman (1966) (36 pts.) } \\ + & \text { Phinney and Humphries (1970) (169 pts.) } \\ * & \text { Arai et al. (1985) (6 pts.) }\end{array}$

FIG. 15: Comparison of the laminar Rayleigh breakup length estimated from equation 12 with $C_{\mathrm{LR}}=8.51$ against experimental data. 
at the time of writing. The $\rho_{\mathrm{g}}^{*}$ approach is essentially empirical and does not apply beyond the cases tested. A more general approach is needed.

Suppose that transition to turbulence on the jet itself (rather than in the nozzle) causes the regime change. Working from that hypothesis, a simple model that fits the available pipe jet data can be derived. I'll start with the assumption that the jet transitions to turbulence when a critical $\operatorname{Re}_{x}$ value is reached, where $x$ is the axial distance from the nozzle - see figure 1 . This suggests that the jet critical Reynolds number is

$$
\operatorname{Re}_{\ell x, \text { trans }} \equiv \frac{\bar{U}_{0} x_{\text {trans }}}{v_{\ell}},
$$

where $x_{\text {trans }}$ is the location of transition. Critical Reynolds number models of transition distance are fairly primitive, but they are simple and to my knowledge they have not been used previously in jet breakup. Note that the jet critical Reynolds number differs from the nozzle critical Reynolds number, Re $e_{\ell, \text { turb }}$. This jet critical Reynolds number presumably is influenced by disturbances present as the jet leaves the nozzle 7 the jet velocity profile, and the ambient gas, and for these reasons the model coefficients used here are not expected to be correct aside from the limiting case of pipe jets with high liquid-gas density ratios (high $\rho_{\ell} / \rho_{\mathrm{g}}$ ). It is also likely that factors which increase the nozzle critical Reynolds number, like reducing flow disturbances, also increase the jet critical Reynolds number.

Transition does not occur in the laminar Rayleigh case, so there $\left.x_{\text {trans }}\right\rangle\left\langle x_{\mathrm{b}}\right\rangle_{\text {LR }}$. When transition does occur, obviously $x_{\text {trans }}\left\langle\left\langle x_{\mathrm{b}}\right\rangle_{\mathrm{LR}}\right.$. The boundary between the laminar Rayleigh and downstream transition cases occurs roughly when the breakup length and the transition length are equal: $x_{\text {trans }}=\left\langle x_{\mathrm{b}}\right\rangle_{\mathrm{LR}}$. This is only an approximate criteria for reasons which will be mentioned shortly. Using equation 12 for $\left\langle x_{\mathrm{b}}\right\rangle_{\mathrm{LR}}$, writing $x_{\text {trans }}=\left\langle x_{\mathrm{b}}\right\rangle_{\mathrm{LR}}$ returns

$$
\frac{v_{\ell} \operatorname{Re}_{\ell x, \text { trans }}}{\bar{U}_{0}}=d_{0} \cdot C_{\mathrm{LR}}\left(\mathrm{We}_{\ell 0}^{1 / 2}+3 \frac{\mathrm{We}_{\ell 0}}{\operatorname{Re}_{\ell 0, \text { crit }}}\right),
$$

which can be rewritten as

$$
\operatorname{Re}_{\ell 0, \text { crit }}=\frac{\operatorname{Re}_{\ell x, \text { trans }}-3 C_{\mathrm{LR}} \mathrm{We}_{\ell 0}}{C_{\mathrm{LR}} \mathrm{We}_{\ell 0}^{1 / 2}} .
$$

The jet critical Reynolds number $\operatorname{Re}_{\ell x \text {, trans }}$ can be found in two different ways. The first is by measuring the transition distance on images, which returns $\operatorname{Re}_{\ell x \text {, trans }}=7.16 \times 10^{4}$ using 8 data points (Grant, 1965 and Rupe, 1962), with a range of $5.61 \times 10^{4}$ to $9.75 \times 10^{4}$. The second is by finding the implied value of $\operatorname{Re}_{\ell x}$, trans from equation 16 , calculated for all data points in the compilation marked as in transition between the laminar Rayleigh and downstream transition regimes. The latter suggests that $\operatorname{Re}_{\ell x \text {,trans }}=1.75 \times 10^{5}$ using 79 data points (Arai et al., 1985; Grant 1965; Phinney, 1973, and Phinney and Humphries, 1970), with a range of $4.75 \times 10^{4}$ to $7.18 \times 10^{5}$.

While the two estimates differ by a factor of 2.44 , there is broad overlap between their distributions. Consequently, the idea of a jet critical Reynolds number determining the regime transition is not discounted by the data. Additionally, the data compilation classifies each data point by

\footnotetext{
${ }^{\ddagger}$ As mentioned in a footnote in $\S 3.4$ even laminar jets have fluctuations, whose strength could presumably be measured with a turbulence intensity.
}

Volume 30, Issue x, 2020 
breakup length trend, not the appearance of transition waves. The two are similar but not identical, and this is why $x_{\text {trans }}=\left\langle x_{\mathrm{b}}\right\rangle_{\mathrm{LR}}$ is only approximate. When turbulence transition begins downstream (i.e., beyond $x_{\text {trans }}=\left\langle x_{\mathrm{b}}\right\rangle_{\mathrm{LR}}$ ), the breakup length does not immediately start decreasing as velocity increases. In fact, with increasing velocity, all else equal, the breakup length will often continue to increase as the velocity increases before starting to decrease. Therefore, one should expect that the implied jet critical Reynolds number would be higher than the actual jet critical Reynolds number, as is observed. More advanced theories than that developed in this work could in principle determine where the breakup length peak occurs rather than where downstream turbulence transition occurs. Given that the implied jet critical Reynolds number has an order of magnitude more data and fits the regime data better, it is used in this work.

Equation 16 is plotted in figures 4 and 5 . When the transition length $x_{\text {trans }}$ is very large, it is unlikely that breakup will be observed in many applications, so despite the jet being strictly in the "downstream transition" regime it will appear to be in the laminar Rayleigh regime. For this reason, a line is drawn in the regime diagrams corresponding to when the transition length is 1000 nozzle diameters. Below that line the curve for the onset of the downstream transition regime is drawn lighter as the downstream transition regime will not be easily observed there.

\subsection{Downstream transition regime}

The defining qualitative (visual) characteristic of the downstream transition regime in this work is the flow (or at least the free surface) being laminar at the nozzle outlet, transitioning to turbulence downstream. The defining quantitative characteristic for the downstream transition regime is the breakup length decreasing as the velocity increases. All images with breakup length data in the data compilation are consistent with these definitions — the caveat mentioned in $\S 4.5$ does not appear in the present photos. While future work is needed, to the best of my knowledge for pipe jets the decrease in the breakup length roughly coincides with downstream transition to turbulences

One non-obvious consequence of this categorization is that apparently none of the data of von Ohnesorge (again, who made the earliest regime diagram) is strictly in the turbulent surface breakup or atomization regimes now. Describing breakup at the highest velocities tested, von Ohnesorge (2019, p. 4) states (translated into English) "The atomization process III starts suddenly. The jet shows a smooth surface in the immediate vicinity of the nozzle outlet with axisymmetric swellings, which degenerate into helical transverse displacements of increasing amplitude." Presumably the "smooth surface" refers to an initially laminar flow. The images provided in the journal article lack the resolution to show that the jet was initially laminar, so we only have von Ohnesorge 's word that the jet was initially laminar. Some recent DNS studies may confirm that the flow at the outlet of a fuel spray nozzle (presumably like the nozzle von Ohnesorge used) is laminar (Agarwal and Trujillo, 2018 and Trujillo et al., 2018), however, this could be an artifact of not specifying turbulent inflow boundary conditions, as neither paper discusses whether turbulence was injected into the computational domain at the inflows.

Note that just because the flow is initially laminar does not mean that the breakup is not vigorous. On the contrary, as Hoyt and Taylor (1985) note, liquid jet flows which are initially laminar but transition downstream can have particularly vigorous breakup. Indeed, as can be

\footnotetext{
${ }^{\S}$ Another possibility is that the boundary layer is laminar but the core of the flow is turbulent. Then, the spread of the turbulent region to the free surface can cause an apparent transition. Pipe jets, studied in this work, will not show this behavior as it typically occurs in a converging nozzle.
} 
seen in figures 12 and 14 , the breakup length in the downstream transition regime can be lower than in the atomization regime. But not all downstream transition regime breakup is as vigorous. I propose that the "downstream transition regime" is actually a class of regimes. One of these regimes is like the Rayleigh regime (see figure 7), another is like the turbulent surface breakup regime (see figure 9), and another is like the atomization regime (see figure 8 The turbulent surface breakup and atomization regimes as defined in this work could be viewed as merely fully turbulent versions of those regimes, while their corresponding regimes in the downstream transition class are merely transitional versions.

Unfortunately, because pipe nozzles have low critical Reynolds numbers, the downstream transition part of the $\mathrm{We}_{\ell 0}-\mathrm{Re}_{\ell 0}$ parameter space as seen in figure 5 is too small to easily distinguish between the different varieties of downstream transition breakup. Studies into the downstream transition regime in the future should use nozzles with higher nozzle critical Reynolds numbers for this reason.

From a modeling perspective, it is necessary to consider turbulence transition. The jet behaves differently before, during, and after transition. In principle, one could model the laminarto-turbulent transition by changing the behavior of the jet (which could be the growth rate in a linear stability theory like that of Weber (2019)) when the transition length $x_{\text {trans }}$ is reached. This model contrasts strongly with the prevailing models in the literature. Typical models for this regime (those discussed in $\$ 4.5$ ) assume that the growth rate does not change as a disturbance propagates downstream. This is false in the downstream transition regime - see figure 8 for an example. In figure 8 , the disturbance on the jet is small until it starts growing rapidly after what appears to be turbulence transition. Non-linear theories could avoid the constant growth rate approximation, however, it may be sufficient and easier to use a linear model with different behavior before and after transition and possibly a jump in the disturbance at transition. Presumably the turbulence intensity increases dramatically as the velocity increases in the downstream transition regime.

Given the present lack of accurate models for this regime, the breakup length model used for the downstream transition regime in figures 12 and 14 is

$$
\frac{\left\langle x_{\mathrm{b}}\right\rangle}{d_{0}} \propto \mathrm{We}_{\ell 0}^{-1},
$$

with the constant of proportionality chosen by setting the breakup length of the laminar Rayleigh regime equal to equation 17 at the transition point. This model is simple and motivated by the observation of Etzold et al. (2018) that at least sometimes in the downstream transition regime $\left\langle x_{\mathrm{b}}\right\rangle \propto \bar{U}_{0}^{-2}$.

The droplet size is similarly uncertain. Convention suggests a representative droplet size in this regime is comparable to the nozzle outlet diameter, but as can be seen in figures 7 and 8 , the droplet size could vary from similar to on the order of the nozzle outlet diameter to smaller than the nozzle outlet diameter.

\footnotetext{
"It is possible that the breakup observed in figure 8 is entirely independent of aerodynamic effects, which would make the mechanism different than atomization, or possibly there are multiple types of this vigorous breakup, some of which have aerodynamic influence and others which do not.
}

Volume 30, Issue $x, 2020$ 


\subsection{Transition to turbulence inside the nozzle}

Depending on the location in the regime diagram, once transition is complete, transition to turbulence could decrease the breakup length (e.g., figure 13, transition from laminar to turbulent Rayleigh, as shown by Mansour and Chigier (1994a, fig. 5)), or increase it (e.g., figures 12 and 14, transition from downstream transition to turbulent surface breakup or atomization, as suggested by Hoyt and Taylor (1985)). The latter is because the downstream transition regime can be particularly unstable, as seen through it's decreasing breakup length with increasing velocity. Hoyt and Taylor (1985) recommend that if one wants a more stable jet to bring turbulence transition inside of the nozzle if it is occurring outside of the nozzle. This seems counterintuitive if you believe that earlier transition is bad, but one way to rephrase this recommendation is to avoid the downstream transition regime if you want a stable jet. Even the atomization regime would be preferable in terms of the breakup length.

See $\S 3.4$ for a discussion of the nozzle critical Reynolds number for turbulence.

\subsection{Turbulent Rayleigh regime}

As stated earlier, the turbulent Rayleigh regime appears similar to the Rayleigh regime, except that the jet is now turbulent at the nozzle outlet. An image of a jet in the turbulent Rayleigh regime is shown in figure 10. Compared against the laminar Rayleigh regime, the breakup in the turbulent Rayleigh regime is less regular, but large droplets are still being produced downstream like in the laminar Rayleigh regime. The turbulent Rayleigh regime follows Weber's theory for the breakup length (equation 12), with the Reynolds number variation neglected here as it is negligible at high Reynolds numbers:

$$
\frac{\left\langle x_{\mathrm{b}}\right\rangle}{d_{0}}=C_{\mathrm{TR}} \mathrm{We}_{\ell 0}^{1 / 2} .
$$

The value of $C_{\mathrm{TR}}$ is lower than $C_{\mathrm{LR}}$. Fitting a model with a constant value of $C_{\mathrm{TR}}$ to the available pipe jet data returns $C_{\mathrm{TR}}=2.79$, however, the $R^{2}$ value is only 0.641 . The constant $C_{\mathrm{TR}}$ model has a lower $R^{2}$ value compared against the laminar case because $C_{\mathrm{TR}}$ varies appreciably with the liquid Weber number. It is anticipated that $C_{\mathrm{TR}}$ also varies with the turbulence intensity. The available pipe jet data in this regime has little variation in the turbulence intensity, so the sensitivity to the turbulence intensity can not be determined from the data compilation in this work. This sensitivity will be estimated through a simple model.

Typically, free surface disturbance amplitudes in temporal stability theory are modeled as following exponential growth (Weber. 2019):

$$
\delta=\delta_{0} \exp \left(\omega_{\mathrm{m}} t\right)
$$

where $\delta$ is the free surface radial perturbation amplitude, $\delta_{0}$ is the initial disturbance level (dimension of length), $\omega_{\mathrm{m}}$ is the growth rate of the disturbance, and $t$ is the time since the jet exited the nozzle. This assumption implies that the coefficient on the breakup length $\left(C_{\mathrm{LR}}\right.$ or $\left.C_{\mathrm{TR}}\right)$, in the typical notation) is independent of the Weber number. This is incorrect in the turbulent Rayleigh case. By hypothesis, the initial free surface deformation in the turbulent Rayleigh case is zero - this would mean that $\delta_{0}=0$ and that $\delta=0$ for all times in equation 19 . However, equation 19 is only one of many possible solutions to the stability problem, which differ in their initial conditions. This has been noted by previous researchers (García and González, 2008, p. 5). Equation 19 suggests not only that the initial disturbance amplitude is $\delta_{0}$ but also suggests there 
is a non-zero initial disturbance velocity $\delta_{0}^{\prime}$. The way the theory is typically presented implies that only an amplitude is set, but as $\delta$ is the solution of a second-order differential equation, two initial conditions are required. In the laminar Rayleigh case, velocity fluctuations are presumably small and the disturbance at the nozzle is likely caused by geometric imperfections. Using only an initial disturbance height seems reasonable in this case, i.e., $\delta_{0}>0, \delta_{0}^{\prime} \approx 0$. Consequently the choice of equation 19 can be seen to be made more for convenience than realism in the laminar Rayleigh case ${ }^{\|}$For turbulent Rayleigh, however, velocity fluctuations are appreciable and geometric imperfections are relatively unimportant, so $\delta_{0} \approx 0$ and $\delta_{0}^{\prime}>0$. Because the turbulence decays downstream in this regime (Mansour and Chigier, 1994b, fig. 18), the impact of turbulent velocity fluctuations at the surface is felt primarily near the nozzle, so it is reasonable to model the effect of the disturbances only in the initial condition in the turbulent Rayleigh regime this is not expected to extend to other regimes.

To set both $\delta_{0}=0$ and $\delta_{0}^{\prime} \neq 0$ requires at least two modes. For the inviscid case considered here, it can be shown that the most unstable mode's growth rate, $\omega_{\mathrm{m}}$, has a corresponding decay rate, $-\omega_{\mathrm{m}}$. The decay rate in the viscous case is not simply the negative of the growth rate. The inviscid case simplifies the math appreciably and is realistic for turbulent Rayleigh, so it will be used here. Now, instead of equation 19 the disturbance amplitude evolves according to

$$
\delta=\delta_{1} \exp \left(\omega_{\mathrm{m}} t\right)+\delta_{2} \exp \left(-\omega_{\mathrm{m}} t\right)
$$

where $\delta_{1}$ and $\delta_{2}$ are arbitrary constants. For the initial conditions $\delta_{0}=0$ and $\delta_{0}^{\prime} \neq 0$, equation 20 can be written in terms of the hyperbolic sine function:

$$
\delta=\frac{\delta_{0}^{\prime}}{\omega_{\mathrm{m}}} \sinh \left(\omega_{\mathrm{m}} t\right)
$$

To find the breakup length from equation 21, find the time, $t_{\mathrm{b}}$, where the surface disturbance amplitude grows to $d_{0} / 2$. At that point the surface wave valleys will meet, leading to the breakup of the jet's core. The result is

$$
\frac{\left\langle x_{\mathrm{b}}\right\rangle}{d_{0}}=\operatorname{arccsch}\left(\frac{2 \delta_{0}^{\prime}}{\bar{U}_{0}} \mathrm{We}_{\ell 0}^{1 / 2}\right) \mathrm{We}_{\ell 0}^{1 / 2},
$$

which implies that

$$
C_{\mathrm{TR}}=\operatorname{arccsch}\left(\frac{2 \delta_{0}^{\prime}}{\bar{U}_{0}} \mathrm{We}_{\ell 0}^{1 / 2}\right),
$$

where arccsch is the inverse hyperbolic cosecant function.

At this point a choice needs to be made for the initial disturbance velocity, $\delta_{0}^{\prime}$. By hypothesis, turbulent fluctuations cause the initial surface deformation. Considering $C_{\mathrm{TR}}$ to be an average over each realization. As $\delta$ is the amplitude of the waves, $\delta$ can not be negative. Consequently, to model the initial disturbance velocity I take the absolute value of the velocity fluctuation, so for

"Performing the same analysis as in the turbulent Rayleigh case for the laminar Rayleigh case leads to $C_{\mathrm{LR}}=\operatorname{arcsech}\left(\frac{2 \delta_{0}}{d_{0}}\right)$ instead of the typical $\ln \left(\frac{d_{0}}{2 \delta_{0}}\right)$, where arcsech is the inverse hyperbolic secant function. Note that the arcsech result is for an inviscid jet but is expected to be reasonable for a viscous jet as well. If $C_{\mathrm{LR}}$ is constant than so is $\delta_{0}$ regardless of whether arcsech or ln are used, though the implied values of $\delta_{0}$ would differ.

Volume 30, Issue $x, 2020$ 
a particular realization, $\delta_{0}^{\prime}=\left|v_{0}\right|$, where $v_{0}$ is the fluctuating radial velocity at the free surface near the nozzle outlet:

$$
C_{\mathrm{TR}}=\left\langle\operatorname{arccsch}\left(\frac{2\left|v_{0}\right|}{\bar{U}_{0}} \mathrm{We}_{\ell 0}^{1 / 2}\right)\right\rangle \approx \operatorname{arccsch}\left(\frac{2\left\langle\left|v_{0}\right|\right\rangle}{\bar{U}_{0}} \mathrm{We}_{\ell 0}^{1 / 2}\right) .
$$

Now, note that

$$
\frac{\left\langle\left|v_{0}\right|\right\rangle}{\bar{U}_{0}} \propto \frac{v_{0}^{\prime}}{\bar{U}_{0}}=\mathrm{Tu}_{0}
$$

The selection $2 \delta_{0}^{\prime}=C_{v} \overline{v_{0}^{\prime}}$ leads to the model

$$
C_{\mathrm{TR}}=\operatorname{arccsch}\left(C_{v} \overline{\mathrm{Tu}}_{0} \mathrm{We}_{\ell 0}^{1 / 2}\right)
$$

The pipe nozzle data from Phinney (1973, 1975) and Sallam et al. (2002) appears to have consistent breakup lengths that can be used to calibrate the model for $C_{\mathrm{TR}}$. Data from Mansour and Chigier (1994a pp. 597-598) was neglected due to inconsistencies with the other data, likely due to using a different definition of the breakup length that examined waves rather than the end of the jet's core (Mansour and Chigier, 1994a, p. 594) Regression analysis on the consistent data suggests that $C_{v}=0.0615$ (31 points, $R^{2}=0.961$ ). The fit with the data is excellent as can be seen in figure 16 The no-slip condition at the nozzle wall would suggest that the initial RMS radial velocity $v_{0}^{\prime}$ is likely much smaller than $\overline{v_{0}^{\prime}}$, so $C_{v}$ is expected to be smaller than 1 , consistent with the data.

Note that the experiments of Vliem (1975, p. 30) with obstructions placed in the nozzle suggest that the breakup length (and as a consequence, the initial disturbance level) in the turbulent Rayleigh regime varies within the measurement uncertainty as the centerline streamwise turbulence intensity increases by a factor of 2.1 from that of a smooth pipe. However, the centerline turbulence intensity is not representative of the near-surface turbulence intensity. The turbulence intensity peaks near the edge of the nozzle/jet. Lissenburg et al. (1975) provides the turbulence intensity at the centerline and at $r / r_{0}=0.9$ for a system geometrically identical to that of Vliem's higher turbulence case at the same Reynolds number. The lower turbulence intensity case for comparison is a smooth pipe, and smooth pipe data from Loulou et al. (1997. fig. 3.16) is at a Reynolds numbers similar to Vliem's experiment. At $r / r_{0}=0.9$, the turbulence intensity of the high turbulence case is only approximately 1.2 times that of a smooth pipe. Assuming that $\overline{\mathrm{Tu}}_{0}$ increases by the same factor, $C_{\mathrm{TR}}$ as calculated by equation 26 for the high turbulence intensity case is found to be 0.95 times its value for a corresponding smooth pipe nozzle. This is within the measurement uncertainty, so Vliem's results do not contradict the $C_{\mathrm{TR}}$ model presented in this work.

The droplet size in the turbulent Rayleigh regime is similar to that of the laminar Rayleigh regime (equation 13). Mansour and Chigier (1994a p. 600) note that the droplet size distribution is bimodal, with the fundamental peak at approximately $D=2.4 d_{0}$, near the value predicted by Weber's theory, and a second peak at $D=0.5 d_{0}$ for satellite droplets. Vliem (1975) gives a

** Data from Mansour and Chigier (1994a) was used for the laminar Rayleigh regime, however, as this data is consistent with that of other researchers despite the difference in definition. Mansour and Chigier (1994a p. 594) note that under their definition the laminar jet breakup length was unambiguous, but note difficulties with the turbulent jet breakup length, so ultimately their definition may not be appropriate for turbulent jets unlike the average definition used in this work. 


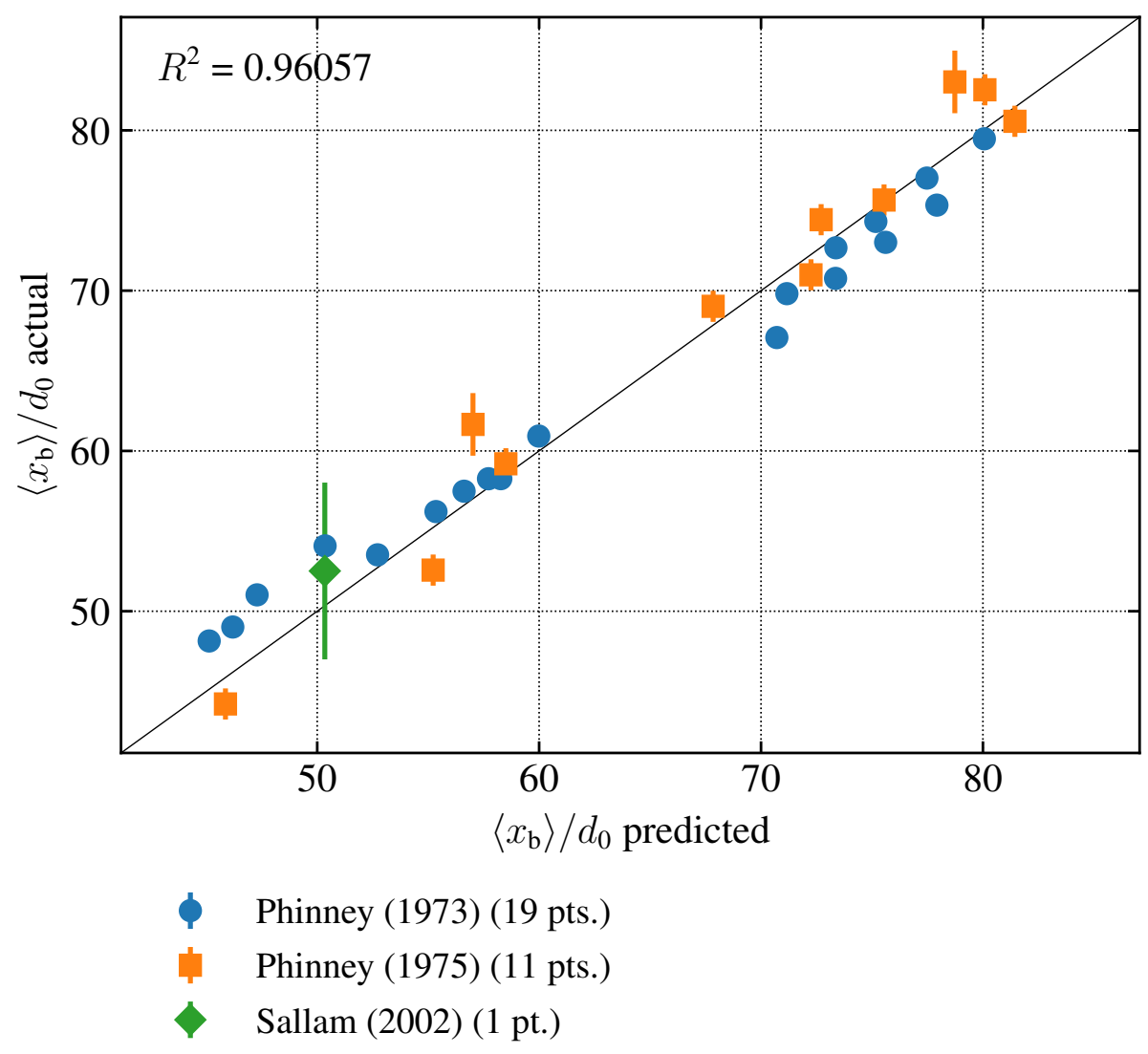

FIG. 16: Comparison of the turbulent Rayleigh breakup length estimated from equation 12 with $C_{\mathrm{TR}}$ modeled with equation 26 against selected experimental data; see text for discussion.

Volume 30, Issue x, 2020 
similar distribution function, albeit with much more frequent satellite droplets. The (fundamental peak) droplet size was not explicitly given by Vliem but was reported by Sterling and Abbott (1981. p. I-4.6) as within $2 \%$ of Weber's theory in Vliem's experiments. The droplet size is expected to be independent of the turbulence intensity in the turbulent Rayleigh regime. This is because the droplet size in Weber's theory is independent of the initial disturbance level - see equation 13

\subsection{Transition from the turbulent Rayleigh regime to the turbulent surface breakup regime}

The transition from the turbulent Rayleigh regime to the turbulent surface breakup regime is gradual, as both the Rayleigh (core breakup) and turbulent surface breakup mechanisms are present in both regimes. The difference is that the Rayleigh mechanism dominates in the turbulent Rayleigh regime, and correspondingly, turbulent fluctuations directly causing breakup at the free surface dominate in the turbulent surface breakup regime. One simple way to measure the relative strengths of each mechanism would be to compute the ratio of the average breakup onset location, $\left\langle x_{\mathrm{i}}\right\rangle$, to the average breakup length in the turbulent Rayleigh regime, $\left\langle x_{\mathrm{b}}\right\rangle$. In the turbulent Rayleigh regime, presumably a long distance is needed for turbulent surface breakup to start, hence, $\left\langle x_{\mathrm{i}}\right\rangle \gg\left\langle x_{\mathrm{b}}\right\rangle$. The opposite is true in the turbulent surface breakup regime. Setting the ratio of the average breakup onset location theory developed by Trettel (2020a) and Weber's breakup length theory (equation 12 using $C_{\mathrm{TR}}$ from equation 26 to a critical value returns

$$
\frac{\left\langle x_{\mathrm{i}}\right\rangle}{\left\langle x_{\mathrm{b}}\right\rangle} \propto \frac{\overline{\mathrm{Tu}}_{0}^{-3} \mathrm{We}_{\ell 0}^{-1}}{\operatorname{arccsch}\left(C_{v} \overline{\mathrm{Tu}}_{0} \mathrm{We}_{\ell 0}^{1 / 2}\right) \mathrm{We}_{\ell 0}^{1 / 2}} \equiv \frac{1}{\widehat{C}_{\mathrm{TR} \text { to TSB }}}
$$

The transition between the two regimes is defined here as a certain critical value of the ratio $\left\langle x_{\mathrm{i}}\right\rangle /\left\langle x_{\mathrm{b}}\right\rangle$. Rearranging this ratio returns

$$
\widehat{C}_{\mathrm{TR} \text { to TSB }}=\operatorname{arccsch}\left(C_{v} \overline{\mathrm{Tu}}_{0} \mathrm{We}_{\ell 0, \text { crit }}^{1 / 2}\right)\left(\overline{\mathrm{Tu}}_{0} \mathrm{We}_{\ell 0, \text { crit }}^{1 / 2}\right)^{3},
$$

where the Weber number has been labeled as the critical Weber number. This implies that $\overline{\mathrm{Tu}}_{0} \mathrm{We}_{\ell 0, \text { crit }}^{1 / 2}$ is a constant, e.g.:

$$
C_{\mathrm{TR} \text { to TSB }}=\overline{\mathrm{Tu}}_{0} \mathrm{We}_{\ell 0, \text { crit }}^{1 / 2} \text {. }
$$

Written explicitly in terms of the critical Weber number, the equation

$$
\mathrm{We}_{\ell 0, \text { crit }}=8 \overline{\mathrm{Tu}}_{0}^{-2}
$$

fits the limited available data.

\subsection{Turbulent surface breakup regime}

Breakup in the turbulent surface breakup regime is caused primarily by turbulent fluctuations perforating the free surface (Trettel, 2020a). Figure 11 shows what a jet in the turbulent surface breakup regime looks like near the nozzle. The breakup here is rather vigorous, and the breakup 
is mainly at the free surface of the jet rather than of the core itself as in the turbulent Rayleigh regime.

A variety of regressions were developed for the turbulent surface breakup regime in this work. The most important, the breakup length regression, made use of data from Kusui (1969) with significant turbulence intensity variation $\left(5.4 \% \leq \overline{\mathrm{Tu}}_{0} \leq 12.7 \%\right)$, among other studies. This regression is (193 points, $R^{2}=0.958$ ):

$$
\frac{\left\langle x_{\mathrm{b}}\right\rangle}{d_{0}}=3.61 \overline{\mathrm{Tu}}_{0}^{-0.275} \mathrm{We}_{\ell 0}^{0.334}
$$

The regression above neglected $\operatorname{Re}_{\ell 0}$ and $\rho_{\ell} / \rho_{\mathrm{g}}$. Including $\operatorname{Re}_{\ell 0}$ and $\rho_{\ell} / \rho_{\mathrm{g}}$, their exponents would be 0.0195 and 0.0171 respectively, nearly zero. This indicates that the influence of these variables is negligible in the turbulent surface breakup regime. The lack of a density ratio influence makes the earlier name for this regime, the "second wind-induced regime", a misnomer, as mentioned in $\S 3.3$. For simplicity the Reynolds number and density ratio were removed from the regression.

Plots comparing equation 31 against experimental data are shown in figures 17 and 18 . Note that the regression was made only against data with breakup lengths measured via electrical conductivity as the large uncertainties seen in the breakup length measurements based on images appreciably change the turbulence intensity exponent, and this change is likely spurious. However, the comparison is against all available breakup length data in the turbulent surface breakup regime.

The regression was cross-validated with non-pipe breakup length data (Ervine et al., 1980 and McKeogh and Elsawy, 1980), excluding points estimated to be in the atomization regime by equation 37. This alternative data set has lower turbulence intensity $\left(0.3 \% \leq \mathrm{Tu}_{\mathrm{c} 0} \leq 8.0 \%\right)$ than the turbulent surface breakup regime pipe jets in the database $\left(5.4 \% \leq \overline{\mathrm{Tu}}_{0} \leq 12.7 \%\right)^{\dagger \dagger}$ The fit between the regression and the alternative data is worse ( 79 points, $R^{2}=0.526$ ), but the error appears to be random - see figure 18 and compare against figure 17 for only pipe jets. The error possibly is due to variables not considered in the regression, e.g., the integral scale and the velocity profile. The error could also be due to Ervine et al. and McKeogh and Elsawy measuring centerline turbulence intensity $\left(\mathrm{Tu}_{\mathrm{c} 0}\right)$ rather than the plane average turbulence intensity $\left(\overline{\mathrm{Tu}}_{0}\right)$ that I use. Another possibility is that the uncertainty of the breakup length is large due to a low number of images used to establish the average - see Trettel (2019) for general uncertainty analysis of the breakup length. Likely all of these are factors. The general agreement suggests that the regression may be valid for $\overline{\mathrm{Tu}}_{0}$ outside its calibration data and consequently may be a useful model for non-pipe jets.

A regression was made for the spray angle in the turbulent surface breakup regime. Analysis of available data in either the turbulent surface breakup or atomization regimes suggests that the spray angle is far too noisy to naively use for regression purposes. This is likely due to the lack of standard definitions of the spray angle - see Trettel (2019) for a discussion of this problem. Additionally, the only study with spray angle data with appreciable turbulence

${ }^{\dagger \dagger}$ While equation 30 suggests much of this data is in the turbulent Rayleigh regime, equation 30 fits data at the higher turbulence intensities mentioned previously, and may not apply for the low turbulence intensities in the alternative data. It is assumed that all of the alternative data is in the turbulent surface breakup regime, as the two experiments are similar and Ervine et al. (1980 §23) suggests that the jets has "intense roughness" which made measuring the breakup length via images difficult. Jets in the turbulent Rayleigh regime are unlikely to be that rough.

Volume 30, Issue $x, 2020$ 


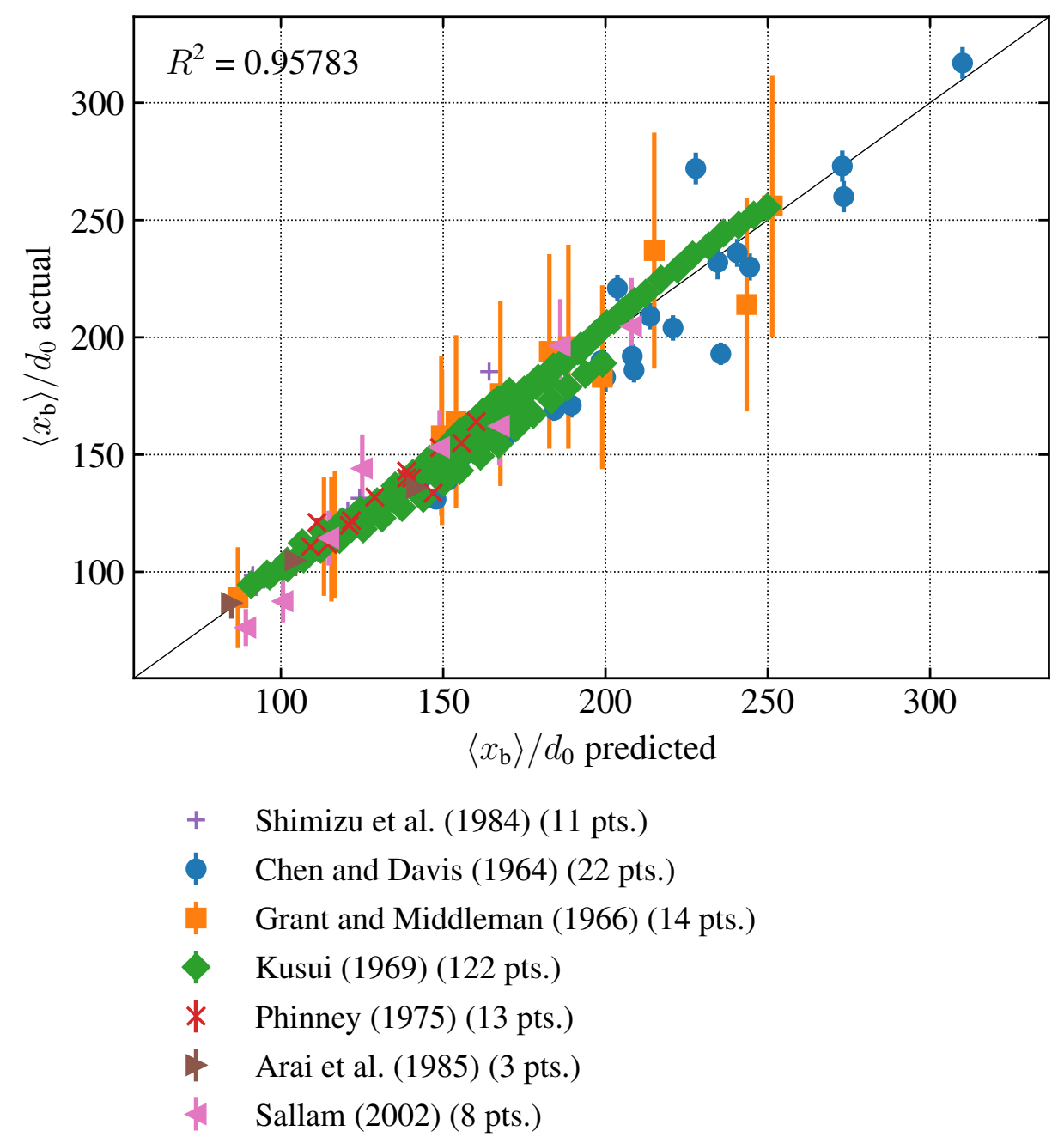

FIG. 17: Comparison of the breakup length regression (equation 31 against experimental data with (estimated) uncertainties. 


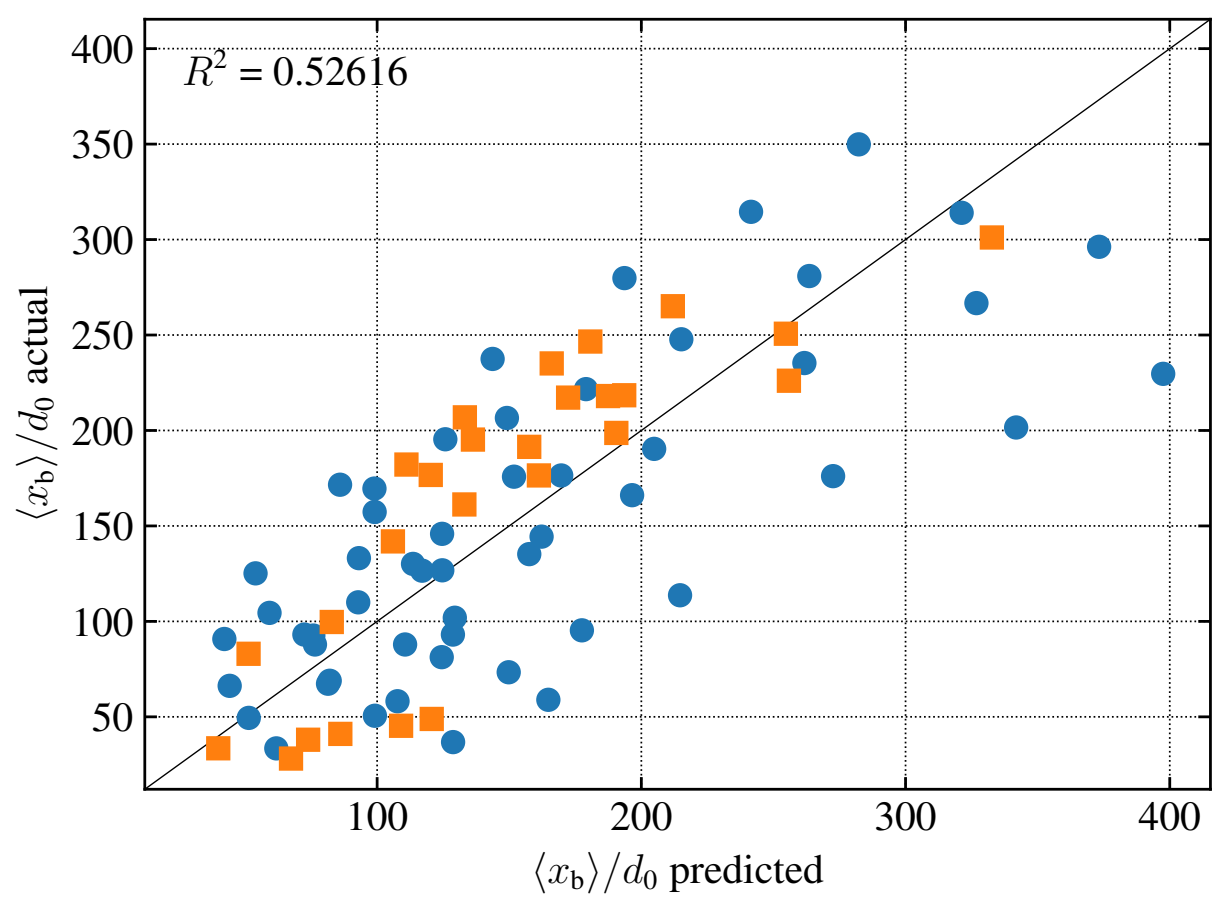

- Ervine et al. (1980) (53 pts.)

McKeogh and Elsawy (1980) (26 pts.)

FIG. 18: Comparison of the breakup length regression (equation 31 against alternative experimental data. 
intensity variation (Skrebkov, 1966) is in the atomization regime (according to equation 37), so it is not strictly possible to determine the sensitivity to the turbulence intensity for the spray angle in the turbulent surface breakup regime at the moment. However, a power law regression was applied to Skrebkov's data and it was assumed that the turbulence intensity exponent in the atomization regime is equal to that of the turbulent surface breakup regime. Then, with the prescribed turbulence intensity variation, another regression was made using only data from the Faeth group (Ruff, 1990 and Sallam, 2002), which appears to be less noisy and may have used a consistent definition, returning (5 points, $R^{2}=0.983$ ):

$$
\tan \left(\frac{\theta_{\mathrm{i}}}{2}\right)=4.73 \times 10^{-4} \overline{\mathrm{Tu}}_{0}^{0.827} \mathrm{We}_{\ell 0}^{0.621} .
$$

Due to the sparsity of consistent data available for the spray angle and high match with the data, for brevity the predicted vs. actual plot is neglected.

Similarly, because there is no pipe jet data with appreciable turbulence intensity variation for $\left\langle x_{\mathrm{i}}\right\rangle, D_{32}$, or $\left\langle v_{\mathrm{d}}\right\rangle$, a regression analysis was done using composite variables including both the Weber number and the turbulence intensity as the theory in Trettel (2020a) predicted they'll appear. For the breakup onset location, the regression is (52 points, $R^{2}=0.758$, predicted vs. actual plot in figure 19]

$$
\frac{\left\langle x_{\mathrm{i}}\right\rangle}{d_{0}}=13.0\left(\overline{\mathrm{Tu}}_{0}^{3} \mathrm{We}_{\ell 0}\right)^{-0.915} .
$$

For the Sauter mean diameter, only data at the breakup onset location had known turbulence intensities, so only data there was used in the regression. Because of confounding between $\overline{\mathrm{Tu}}_{0}$ and $\mathrm{Re}_{\ell 0} \mathrm{I}$ used solely the variable $\overline{\mathrm{Tu}}_{0}^{2} \mathrm{We}_{\ell 0}$ in the regression analysis, consistent with CDRSV theory (Trettel, 2020a). The regression is (29 points, $R^{2}=0.712$, predicted vs. actual plot in figure 20]

$$
\frac{D_{32}}{d_{0}}=0.564\left(\overline{\mathrm{Tu}}_{0}^{2} \mathrm{We}_{\ell 0}\right)^{-0.644} .
$$

This regression includes only droplets formed at the breakup onset location and does not contain the large droplets formed downstream. As suggested by figure 11, the droplet sizes in the turbulent surface breakup regime are smaller than the nozzle outlet diameter as can be seen in figure 20

And like the Sauter mean diameter, for the radial droplet velocity after formation, only data at the breakup onset location had known turbulence intensities. As such, the regression is (17 points, $R^{2}=-0.0131$, predicted vs. actual plot in figure 21.

$$
\frac{\left\langle v_{\mathrm{d}}\right\rangle}{\overline{v_{0}^{\prime}}}=0.0582\left(\overline{\mathrm{Tu}}_{0}^{2} \mathrm{We}_{\ell 0}\right)^{0.0322} .
$$

Note that the $R^{2}$ value for the droplet velocity is low because of the extremely high uncertainties in the data $-60 \%$ according to $\mathrm{Wu}$ (1992, p. 129). See figure 21. It is unlikely any reasonable model would fit this data well because the data is largely random noise.

A comparison of these regressions to theory is in Trettel (2020a).

\subsection{Atomization regime and the transition from the turbulent surface breakup regime}

A variety of mechanisms are factors in the atomization regime: turbulence, aerodynamic influence of the ambient gas (controlled by the density ratio), cavitation, the velocity profile, and 


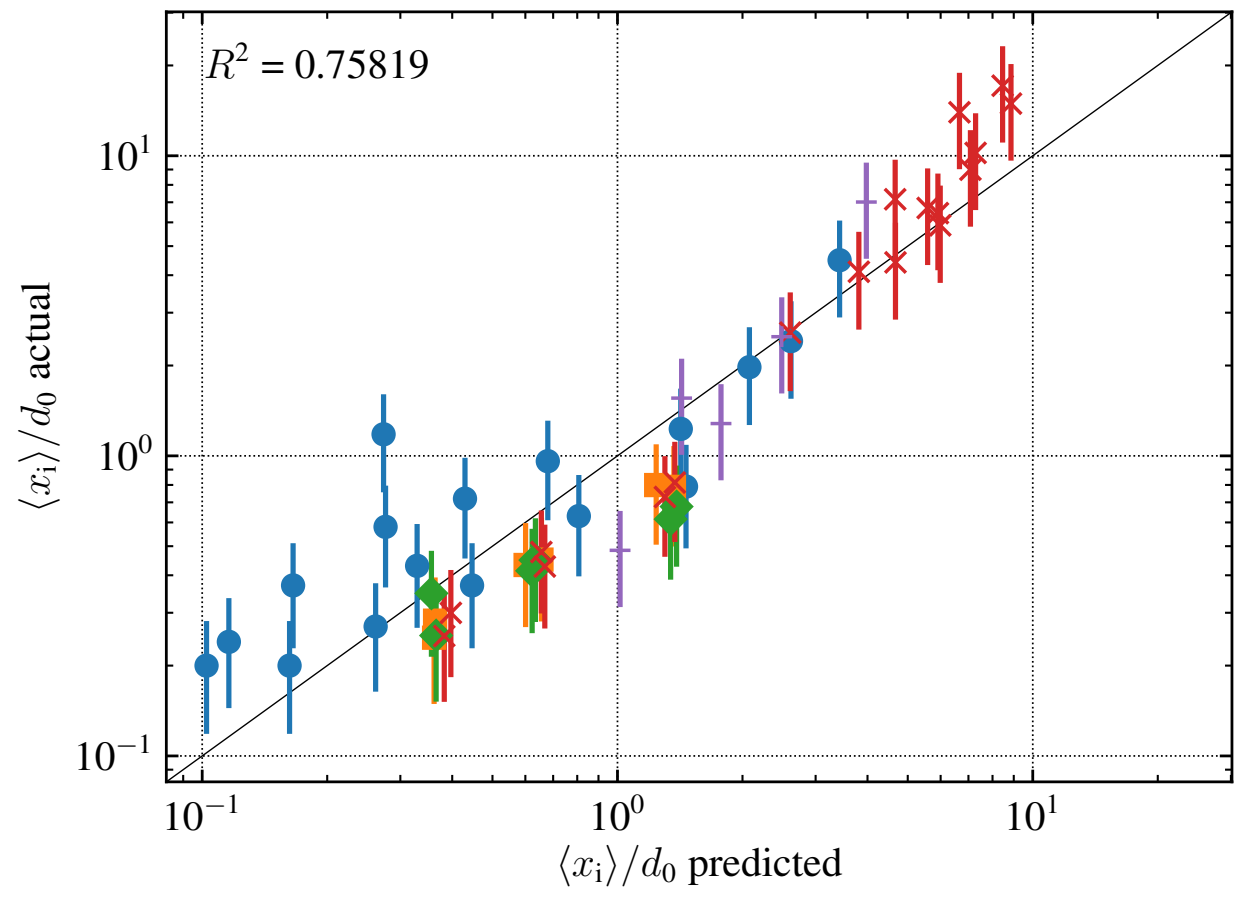

Wu (1992) (17 pts.)

Wu et al. (1995) (6 pts.)

$>\quad$ Wu and Faeth (1993) (6 pts.)

* Wu and Faeth (1995) (18 pts.)

+ Sallam (2002) (5 pts.)

FIG. 19: Comparison of the breakup onset location regression for the turbulent surface breakup regime (equation 33 against experimental data with uncertainties. 


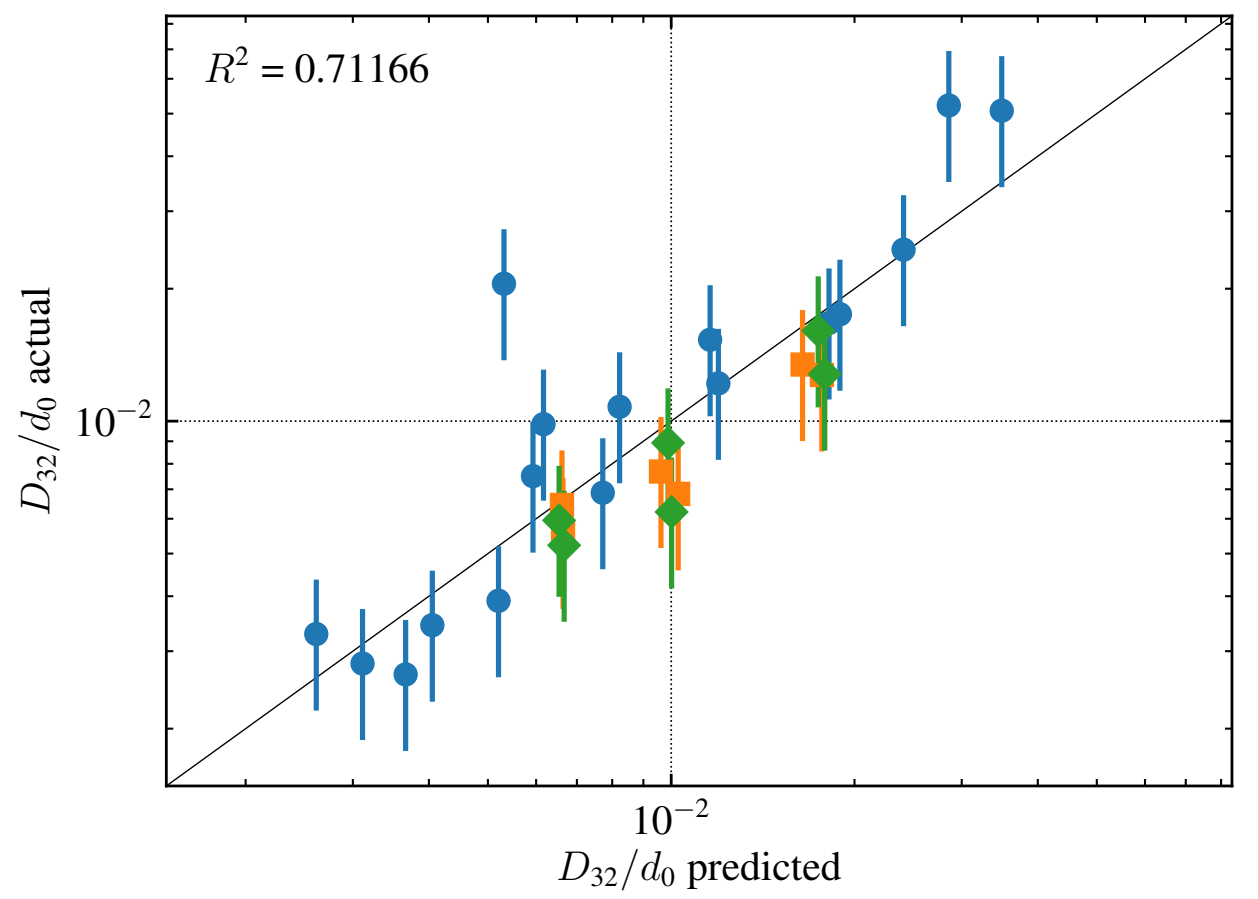

Wu $(1992)$ (17 pts.)

Wu et al. (1995) (6 pts.)

$>$ Wu and Faeth (1993) (6 pts.)

FIG. 20: Comparison of the Sauter mean diameter regression for the turbulent surface breakup regime (equation 34 against experimental data with uncertainties. 


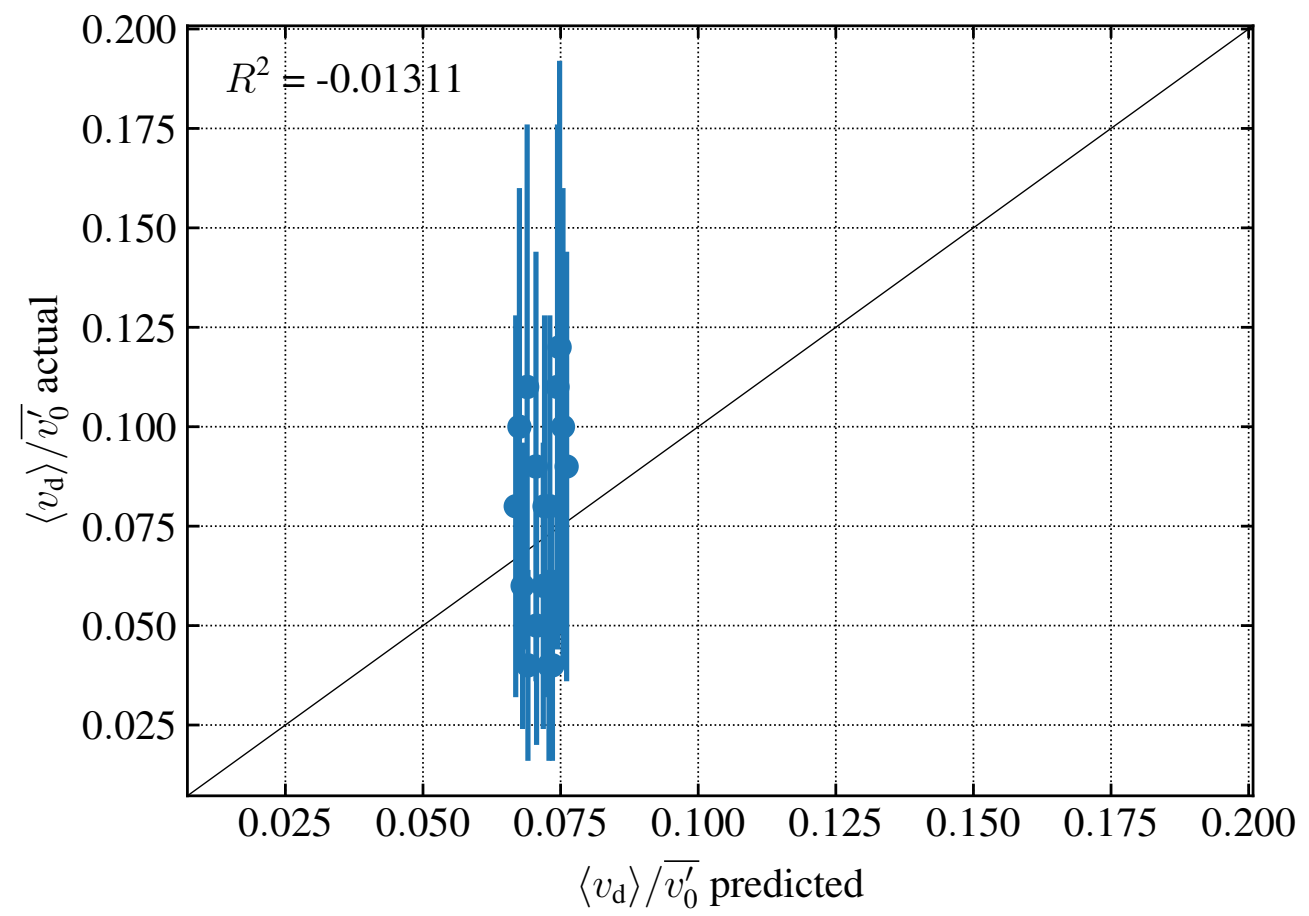

Wu $(1992)(17$ pts.)

FIG. 21: Comparison of the radial droplet velocity after formation regression for the turbulent surface breakup regime (equation 35) against experimental data with uncertainties. 
compressibility. The atomization regime is superficially identical to the turbulent surface breakup regime as shown in figure 11 . This highlights the need for quantitative characteristics defining the atomization regime, in this case using the breakup length. Here I'll define atomization as a regime where the power law increase of the breakup length with the Weber number no longer applies. At low Mach numbers, the breakup length plateaus when the jet enters the atomization regime (Kusui, 1969 and Sallam, 2002). At higher Mach numbers the breakup length peaks before the plateau (Arai et al., 1985 and Shimizu et al., 1984). In this work, data at and surrounding the peak is classified as transitional between the turbulent surface breakup and atomization regimes.

Drawing a line in a regime diagram to get the boundary equation directly does not seem prudent as the data is sparse at high Weber and Reynolds numbers. Alternatively, given the different behaviors of the breakup length in the turbulent surface breakup and atomization regimes, finding the intersection of breakup length regressions for these regimes would return an equation for the boundary between these regimes. Unfortunately, if one limits the regression for the atomization regime to the available low Mach number $\left(\mathrm{Mag}_{\mathrm{g}}<0.3\right)$ data for pipe jets, one can not distinguish between cavitation and density ratio effects. The cases with low $\rho_{\ell} / \rho_{\mathrm{g}}$ also have only sudden contraction entrances to the pipe, while the cases with high $\rho_{\ell} / \rho_{\mathrm{g}}$ have only smooth entrances to the pipe. Sudden contractions are more prone to cavitation (Ahn et al., 2006, fig. 3), which reduces the breakup length, just like low density ratios. Problems like this are called "confounding" and is discussed more in Trettel (2019).

Fitting the available data, shortcomings and all, returns the following equation for the breakup length in the atomization regime (11 points, $R^{2}=0.602, \rho_{\ell} / \rho_{\mathrm{g}}$ ranging from 29.4 to 882 , predicted vs. actual plot in figure 22:

$$
\frac{\left\langle x_{\mathrm{b}}\right\rangle}{d_{0}}=5.31 \overline{\mathrm{Tu}}_{0}^{-0.568}\left(\frac{\rho_{\ell}}{\rho_{\mathrm{g}}}\right)^{0.335},
$$

which implies the following turbulent surface breakup to atomization regime boundary:

$$
\mathrm{We}_{\ell 0, \text { crit }}=3.17 \overline{\mathrm{Tu}}_{0}^{-0.876}\left(\frac{\rho_{\ell}}{\rho_{\mathrm{g}}}\right)^{1.00} .
$$

The density ratio exponent given above is accurate to 3 significant figures - it does not equal 1 exactly here. Note that equation 37 approximates a simple critical gas Weber number criteria:

$$
\mathrm{We}_{\mathrm{g} 0, \text { crit }}=3.17 \overline{\mathrm{Tu}}_{0}^{-0.876} \text {. }
$$

The use of a critical gas Weber number for atomization as suggested by Reitz (1978, p. 8) and discussed in $\S 3.2$ appears reasonable given the limited amount of data available.

Further, the critical gas Weber number predicted by equation 37 for $5 \%$ turbulence and a density ratio of 1000/1.2 (approximating water and air) is 44.8, not far from the number 40.3 Reitz suggested in error. The simplest explanation for why Reitz's criteria ends up being accurate despite the miscalculation is coincidence combined with the fact that the data the criteria was based on did not look at quantitative characteristics like the breakup length. It likely corresponded to a different boundary, assuming it was not entirely spurious.

Finally, given the difficulty of distinguishing between the turbulent surface breakup and atomization regimes based on single images alone, a criteria was developed to allow the regime 


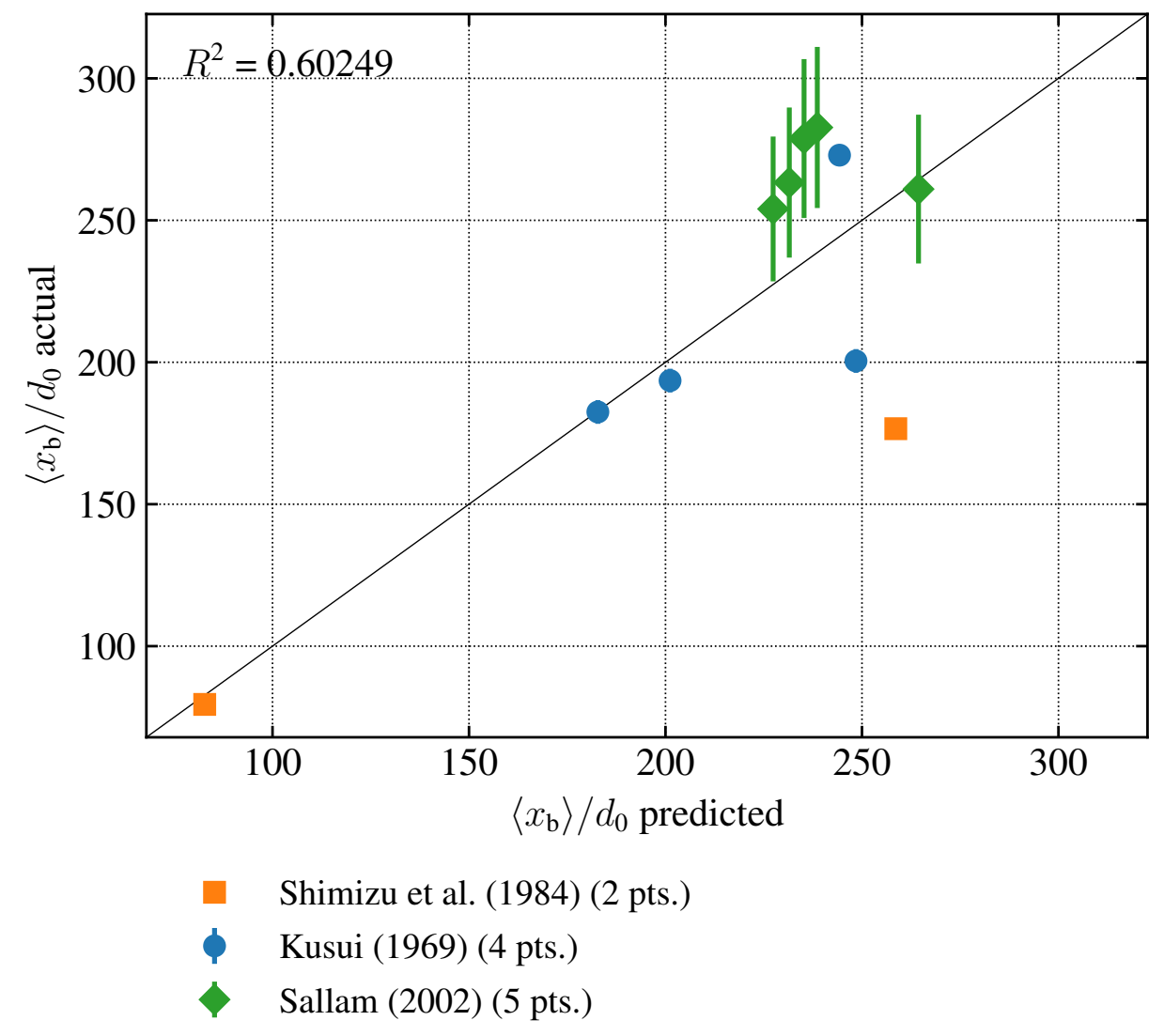

FIG. 22: Comparison of the breakup length regression for the atomization regime (equation 36 against experimental data with (estimated) uncertainties.

Volume 30, Issue $x, 2020$ 
to be estimated based on the spray angle, independent of the Weber number. To develop this criteria, substitute the simplified atomization boundary equation (equation 38) into the equation for the spray angle in the turbulent surface breakup regime (equation 32) to obtain

$$
\tan \left(\frac{\theta_{\mathrm{i}}}{2}\right)_{\text {crit }}=9.69 \times 10^{-4}\left(\frac{\rho_{\ell}}{\rho_{\mathrm{g}}}\right)^{0.621} \overline{\mathrm{Tu}}_{0}^{0.282}
$$

In the data compilation, equation 39 was used to determine the regime for images where the regime was ambiguous between turbulent surface breakup and atomization, as the two are visually similar. The criteria used were as follows: For $\tan \left(\theta_{\mathrm{i}} / 2\right) / \tan \left(\theta_{\mathrm{i}} / 2\right)_{\text {crit }} \leq 0.5$, the regime was marked as turbulent surface breakup. For $0.5<\tan \left(\theta_{i} / 2\right) / \tan \left(\theta_{i} / 2\right)_{\text {crit }} \leq 1.25$, the regime was marked as transitional between turbulent surface breakup and atomization. For $\tan \left(\theta_{i} / 2\right) / \tan \left(\theta_{i} / 2\right)_{\text {crit }}>1.25$, the regime was marked as atomization.

A brief comment on droplet size in the atomization regime: Aerodynamic effects will enhance the formation of droplets by free surface turbulence, making the droplet sizes smaller than in the turbulent surface breakup regime. The reader is referred to the study of $\mathrm{Wu}$ and Faeth (1993) for a detailed experimental treatment of these effects on the droplet size.

\subsection{Universality of the regime diagram}

The reader may be concerned that the new regime diagram applies only for the behavior of the jets in terms of breakup length, and not, for instance, for the visual appearance of the jet or for other quantities like the droplet size. A comparison of regime data classified visually and regime data classified by breakup lengths would be the clearest way to check whether visual and breakup length regimes are consistent. Compare figures 23 and 24 - no major differences appear aside from the fact that the visual data is more sparse.

Other quantities of interest were not studied in detail in this work, so it is possible that regimes classified with other quantities of interest are inconsistent with the new regime diagram.

An argument can be made for broad universality of the regime diagram: A change in the regime changes the physical mechanisms relevant to the problem. The behavior of each quantity of interest is determined by the physical mechanisms involved. Consequently, changing the regime should simultaneously change the behavior of multiple quantities of interest, making the regimes "universal". The most significant flaw to this argument is that even if true, some quantities of interest still do not change across regimes. For example, the droplet size is similar in both the laminar and turbulent Rayleigh regimes. For that reason, regimes classified in terms of droplet sizes may not be able to distinguish between the two Rayleigh regimes.

There is one regime boundary where the visual appearance of the jet and the breakup length trend do not precisely match: the boundary between the laminar Rayleigh and downstream transition regimes. To summarize, the name "downstream transition" suggests that the regime starts when turbulence transition occurs downstream on the jet. However, as velocity increases, downstream turbulence transition occurs slightly before the peak in the breakup length used to demarcate the breakup length regimes. The practical difference between the two definitions is small. See $\$ 4.5$ for details.

Additional studies are needed to test how universal the regime diagram developed in this work is. 

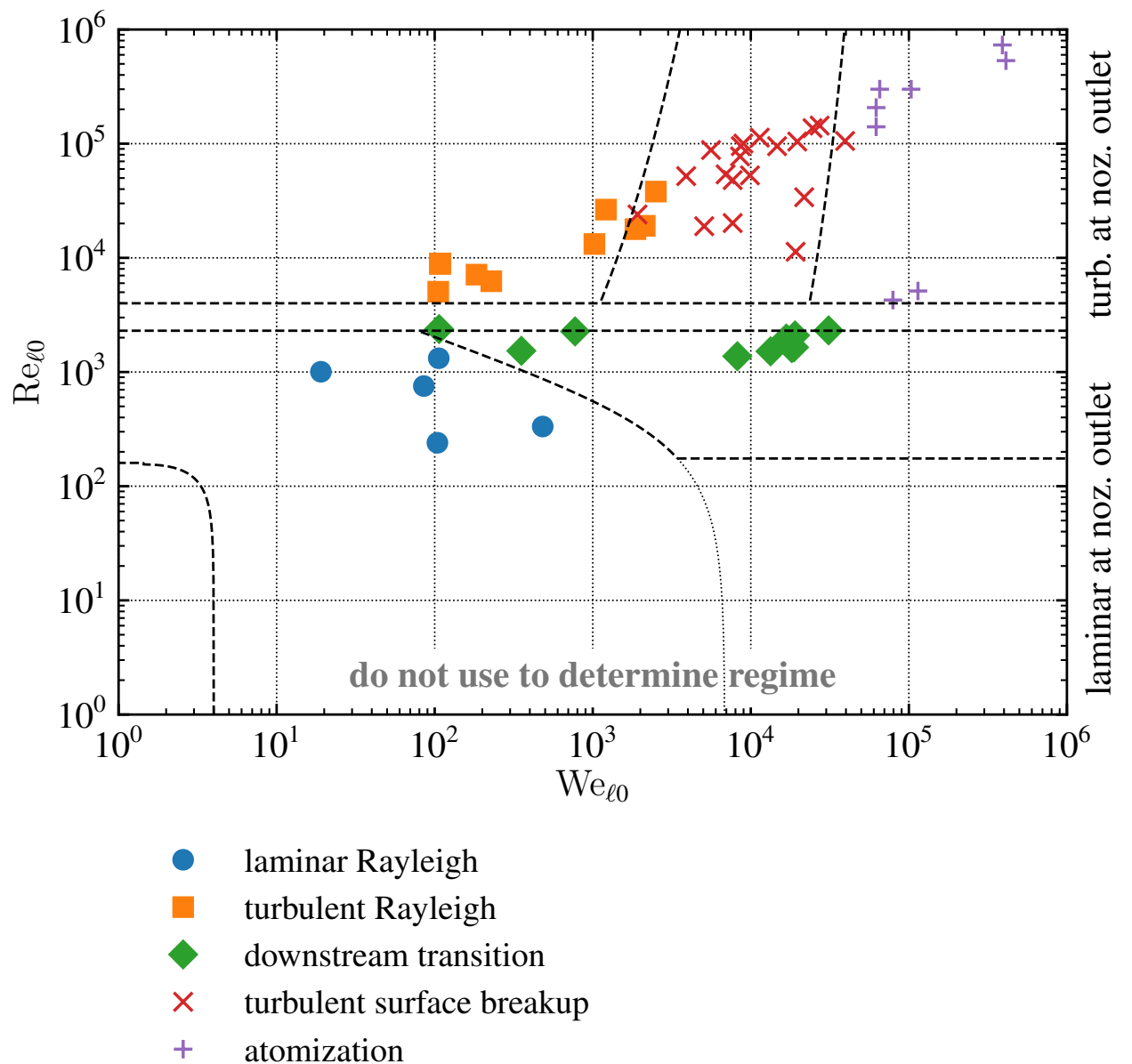

FIG. 23: Regime diagram similar to figure 5 but including only data points where the regime was determined visually. 63 data points. 


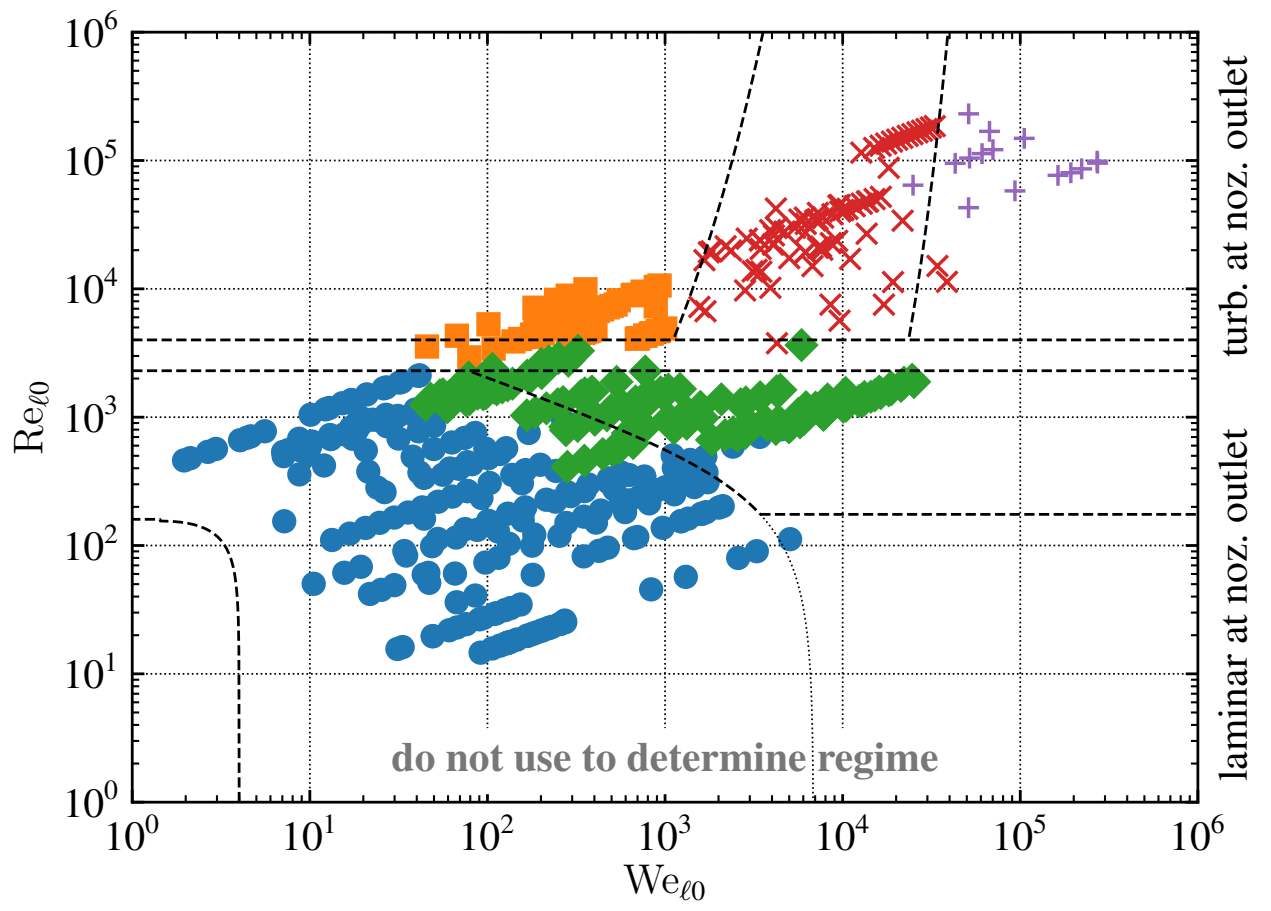

$\begin{array}{ll}- & \text { laminar Rayleigh } \\ & \text { turbulent Rayleigh } \\ \times & \text { downstream transition } \\ \times & \text { turbulent surface breakup } \\ +\quad & \text { atomization }\end{array}$

FIG. 24: Regime diagram similar to figure 5 but including only data points where the regime was determined from breakup length measurements. 514 data points. 


\section{CONCLUSIONS}

Liquid jets break up in 6 regimes recognized in this work: dripping, laminar Rayleigh, downstream transition, turbulent Rayleigh, turbulent surface breakup, and atomization. The turbulent Rayleigh regime has rarely been recognized due to the common but erroneous belief that as the velocity of a jet increases the jet first starts dripping, then enters the (conventionally laminar) Rayleigh regime, then enters the downstream transition regime, then enters the turbulent surface breakup regime, and then enters the atomization regime. This regime progression was shown to be only one of several possibilities. Jets with high Ohnesorge numbers may never enter the turbulent surface breakup regime and skip from downstream transition to atomization. Similarly, jets with low Ohnesorge numbers and low nozzle critical Reynolds numbers may never enter the downstream transition regime and instead enter the turbulent Rayleigh regime prior to the turbulent surface breakup regime.

The regime of a jet is typically determined through qualitative comparison against prototypical images of jets. This procedure was shown to be ambiguous, and instead regime classification based on quantitative characteristics like the breakup length was proposed.

The critical Reynolds number for the onset of turbulence at the nozzle outlet is a factor typically neglected in regime diagrams, but is included in the new regime diagram in this work. The nozzle critical Reynolds number can vary by roughly two orders of magnitude in practice, and whether the jet is turbulent or laminar at the nozzle outlet can strongly influence how the jet breaks up.

The downstream transition regime itself appears to contain multiple other regimes similar each of the turbulent regimes, however, the available data makes studying the boundaries inside of the downstream transition regime difficult. This is due to the low nozzle critical Reynolds number in the data used in this study, which made the area in the $\mathrm{We}_{\ell 0}-\mathrm{Re}_{\ell 0}$ parameter space covered by the downstream transition regime too small to map the regime. New studies with higher and quantified nozzle critical Reynolds numbers are needed to study the downstream transition class of regimes.

The popular Ohnesorge diagram (figure 2) and more recent variations of it (figure 3 ) are inaccurate and should not be used. A schematic diagram (figure 4) is proposed as a replacement, though it must be emphasized that this diagram is merely a schematic which applies only for a special case. More general equations for the boundaries of each regime were given to determine the regime in more general cases.

\section{ACKNOWLEDGMENTS}

Dr. Andrew Trettel carefully read this paper and made thoughtful suggestions. Dr. O. A. Ezekoye emphasized the inaccuracy of qualitative regime classification and this prompted me to do a small poll into the reliability of qualitative regime classification, which was not included in this work due to brevity. Dr. Mathias Etzold is thanked for improving $\S 3.2$. Henk Vliem is thanked for providing a copy of Vliem (1975). An anonymous reviewer is thanked for suggestions which improved the terminology in and presentation of this paper. Michael Ravnitzky is thanked for proofreading this paper and improving its clarity.

\section{REFERENCES}

Agarwal, A. and Trujillo, M.F., A Closer Look at Linear Stability Theory in Modeling Spray Atomization, International Journal of Multiphase Flow, vol. 109, pp. 1-13, 2018.

Volume 30, Issue x, 2020 
Ahn, K., Kim, J., and Yoon, Y., Effects of orifice internal flow on transverse injection into subsonic crossflows: Cavitation and hydraulic flip, Atomization and Sprays, vol. 16, no. 1, pp. 15-34, 2006.

Arai, M., Shimizu, M., and Hiroyasu, H., Break-up length and spray angle of high speed jet, Proceedings of the 3rd International Conference on Liquid Atomisation and Spray Systems, Eisenklam, P. and Yule, A. (Eds.), Institute of Energy, Imperial College, London, UK, pp. IB/4/1-IB/4/10, 1985.

Asset, G.M. and Bales, P.D., Hydraulic Jets at Low Reynolds Number and Constant Weber Number, Medical Laboratories Research Report 69, Chemical Corps Medical Laboratories, Army Chemical Center, Maryland, 1951.

Batchelor, G.K. and Proudman, I., The Effect of Rapid Distortion of a Fluid in Turbulent Motion, The Quarterly Journal of Mechanics and Applied Mathematics, vol. 7, no. 1, pp. 83-103, 1954.

Betchov, R. and Clutter, D.W., On The Breakup Of Liquid Jets, Summary Progress Report 1, George Washington University, Washington, DC, 1955.

Birouk, M. and Lekic, N., Liquid jet breakup in quiescent atmosphere: A review, Atomization and Sprays, vol. 19, no. 6, pp. 501-528, 2009.

Brenn, G. and Stelter, M., A generalized Ohnesorge nomogram for liquid jet breakup regimes, Atomization and Sprays, vol. 30, no. 3, pp. 213-238, 2020.

Chen, T.F. and Davis, J.R., Disintegration of a turbulent water jet, Journal of the Hydraulics Division, vol. 90, no. 1, pp. 175-206, 1964.

Chigier, N. and Reitz, R.D., 1996. Regimes of Jet Breakup and Breakup Mechanisms (Physical Aspects). Recent Advances in Spray Combustion: Spray Atomization and Drop Burning Phenomena. American Institute of Aeronautics and Astronautics, pp. 109-135.

Clanet, C. and Lasheras, J.C., Transition from dripping to jetting, Journal of Fluid Mechanics, vol. 383, pp. 307-326, 1999.

Dumouchel, C., On the experimental investigation on primary atomization of liquid streams, Experiments in Fluids, vol. 45, no. 3, pp. 371-422, 2008.

Eisenklam, P. and Hooper, P.C., Flow characteristics of laminar and turbulent jets of liquid, Report J.R.L. 42, Imperial College of Science and Technology, London, UK, 1958.

Ervine, D.A., McKeogh, E., and Elsawy, E.M., Effect of Turbulence Intensity on the rate of Air Entrainment by Plunging Water Jets, Proceedings of the Institution of Civil Engineers, vol. 69, no. 2, pp. 425-445, 1980.

Etzold, M., Zeitmodulierte, monodisperse Tropfengenerierung zur Herstellung von Feinstsprays [Timemodulated, monodisperse droplet generation for the production of ultra-fine sprays], Dr.-Ing. dissertation, Friedrich-Alexander-Universität Erlangen-Nürnberg, Erlangen, Germany, 2019.

Etzold, M., Deswal, A., Chen, L., and Durst, F., Break-up length of liquid jets produced by short nozzles, International Journal of Multiphase Flow, vol. 99, pp. 397-407, 2018.

Faeth, G.M., Structure and atomization properties of dense turbulent sprays, Symposium (International) on Combustion, vol. 23, no. 1, pp. 1345-1352, 1991.

García, F.J. and González, H., Normal-mode linear analysis and initial conditions of capillary jets, Journal of Fluid Mechanics, vol. 602, pp. 81-117, 2008.

Grant, R.P., Newtonian Jet Stability, PhD dissertation, University of Rochester, Rochester, NY, 1965.

Grant, R.P. and Middleman, S., Newtonian jet stability, AIChE Journal, vol. 12, no. 4, pp. 669-678, 1966.

Haenlein, A., Disintegration of a Liquid Jet, Technical Memorandum 659, National Advisory Committee for Aeronautics, Washington, DC, 1932.

Hoyt, J.W. and Taylor, J.J., Waves on water jets, Journal of Fluid Mechanics, vol. 83, no. 1, pp. 119-127, 1977. 
Hoyt, J.W. and Taylor, J.J., Effect of Nozzle Shape and Polymer Additives on Water Jet Appearance, Journal of Fluids Engineering, vol. 101, no. 3, pp. 304-308, 1979.

Hoyt, J.W. and Taylor, J.J., Pipe-exit flow photography, Cavitation and Polyphase Flow Forum - 1980, ASME, New Orleans, LA, pp. 42-44, 1980.

Hoyt, J.W. and Taylor, J.J., Water-Jet Photography, Naval research reviews, vol. XXXIV, no. 4, pp. 3-9, 1982.

Hoyt, J.W. and Taylor, J.J., Effect of nozzle boundary layer on water jets discharging in air, Jets and Cavities: International Symposium, Kim, J.H., Furuya, O., and Parkin, B.R. (Eds.), Vol. 31 of FED, ASME, Miami Beach, FL, pp. 93-100, 1985.

Kent, J.C. and Brown, G.M., Nozzle Exit Flow Characteristics for Square-edged and Rounded Inlet Geometries, Combustion Science and Technology, vol. 30, no. 1-6, pp. 121-132, 1983.

Kim, S. and Mills, A.F., Condensation on Coherent Turbulent Liquid Jets: Part I — Experimental Study, Journal of Heat Transfer, vol. 111, no. 4, pp. 1068-1074, 1989.

Kroesser, F.W. and Middleman, S., Viscoelastic jet stability, AIChE Journal, vol. 15, no. 3, pp. 383-386, 1969.

Kusui, T., Liquid Jet Flow into Still Gas: Jet Flow by Circular Rough Tube, Bulletin of JSME, vol. 12, no. 53, pp. 1062-1071, 1969.

Lafrance, P., Aiello, G., Ritter, R.C., and Trefil, J.S., Drop spectrometry of laminar and turbulent jets, Physics of Fluids, vol. 17, no. 7, pp. 1469-1470, 1974.

Lebedev, O.N., On the issue of atomizing fuel with diesel injectors, Translation, University of Texas at Austin, 2019.

Lefebvre, A.H. and McDonell, V.G., Atomization and Sprays, 2nd Edition, CRC Press, Boca Raton, FL, 2017.

Lin, S.P. and Reitz, R.D., Drop and Spray Formation from a Liquid Jet, Annual Review of Fluid Mechanics, vol. 30, no. 1, pp. 85-105, 1998.

Lissenburg, R.C.D., Hinze, J.O., and Leijdens, H., An experimental investigation of the effect of a constriction on turbulent pipe flow, Applied Scientific Research, vol. 31, no. 5, pp. 343-362, 1975.

Littaye, G., Contribution à l'étude des jets liquides [Contribution to the study of liquid jets], Publications scientifiques et techniques du Secrétariat d'etat à l'Aviation 181, Paris, France, 1942.

Littaye, G., On a theory for the pulverisation of liquid jets [Review 45], Bulletin, International Institute of Refrigeration, vol. 25, no. II, pp. 57-58, 1944.

Loulou, P., Moser, R.D., Mansour, N.N., and Cantwell, B.J., Direct Numerical Simulation of Incompressible Pipe Flow Using a B-Spline Spectral Method, Technical Memorandum 110436, National Aeronautics and Space Administration, Ames Research Center, Moffett Field, CA, 1997.

Magnotti, G.M., Modeling the Influence of Nozzle-Generated Turbulence on Diesel Sprays, PhD dissertation, Georgia Institute of Technology, Atlanta, GA, 2017.

Malot, H. and Dumouchel, C., Experimental investigation of the drop size distribution of sprays produced by a low-velocity Newtonian cylindrical liquid jet, Atomization and Sprays, vol. 11, no. 3, pp. 227-254, 2001.

Mansour, A. and Chigier, N., Effect of turbulence on the stability of liquid jets and the resulting droplet size distribution, Atomization and Sprays, vol. 4, no. 5, pp. 583-604, 1994a.

Mansour, A. and Chigier, N., Turbulence characteristics in cylindrical liquid jets, Physics of Fluids, vol. 6, no. 10, pp. 3380-3391, 1994b.

McCarthy, M.J. and Molloy, N.A., Review of stability of liquid jets and the influence of nozzle design, The Chemical Engineering Journal, vol. 7, no. 1, pp. 1-20, 1974.

Volume 30, Issue x, 2020 
McKeogh, E.J. and Elsawy, E.M., Air Retained in Pool by Plunging Water Jet, Journal of the Hydraulics Division, vol. 106, no. 10, pp. 1577-1593, 1980.

Miesse, C.C., Correlation of Experimental Data on the Disintegration of Liquid Jets, Industrial \& Engineering Chemistry, vol. 47, no. 9, pp. 1690-1701, 1955.

Mullin, T., Experimental Studies of Transition to Turbulence in a Pipe, Annual Review of Fluid Mechanics, vol. 43, no. 1, pp. 1-24, 2011.

Narasimha, R. and Sreenivasan, K.R., Relaminarization of Fluid Flows, Advances in Applied Mechanics, vol. 19, pp. 221-309, 1979.

Pfenninger, W., 1961. Transition in the inlet length of tubes at high Reynolds numbers, Boundary Layer and Flow Control. Lachmann, G.V. (Ed.). Pergamon, New York, NY, pp. 970-980.

Phinney, R.E., The breakup of a turbulent liquid jet in a gaseous atmosphere, Journal of Fluid Mechanics, vol. 60, no. 4, pp. 689-701, 1973.

Phinney, R.E., Breakup of a turbulent liquid jet in a low-pressure atmosphere, AIChE Journal, vol. 21, no. 5, pp. 996-999, 1975.

Phinney, R.E. and Humphries, W., Stability of a viscous jet - Newtonian liquids, NOLTR 70-5, Naval Ordnance Lab, White Oak, MD, 1970.

Pope, S.B., Turbulent Flows, Cambridge University Press, New York, NY, 2000.

Ranz, W.E., On Sprays and Spraying; a Survey of Spray Technology for Research and Development Engineers, no. 65 in Bulletin, Pennsylvania State University, Department of Engineering Research, University Park, PA, 1956.

Reitz, R.D., Atomization and Other Breakup Regimes of a Liquid Jet, PhD dissertation, Princeton University, Princeton, NJ, 1978.

Reitz, R.D. and Bracco, F.V., 1986. Mechanisms of breakup of round liquid jets. Encyclopedia of Fluid Mechanics. Vol. 3. Gulf Pub. Co., Book Division, Houston, TX, pp. 233-249.

Ruff, G.A., Structure and Mixing Properties of the Near-Injector Region of Nonevaporating PressureAtomized Sprays, PhD dissertation, University of Michigan, Ann Arbor, MI, 1990.

Rupe, J.H., On the dynamic characteristics of free-liquid jets and a partial correlation with orifice geometry, Technical Report 32-207, Jet Propulsion Laboratory, Pasadena, CA, 1962.

Sallam, K.A., Properties of Spray Formation by Turbulent Primary Breakup, PhD dissertation, University of Michigan, Ann Arbor, MI, 2002.

Sallam, K.A., Dai, Z., and Faeth, G.M., Liquid breakup at the surface of turbulent round liquid jets in still gases, International Journal of Multiphase Flow, vol. 28, no. 3, pp. 427-449, 2002.

Schillaci, E., Antepara, O., Balcázar, N., Serrano, J.R., and Oliva, A., A numerical study of liquid atomization regimes by means of conservative level-set simulations, Computers \& Fluids, vol. 179, pp. 137-149, 2019.

Shimizu, M., Arai, M., and Hiroyasu, H., Measurements of Breakup Length in High Speed Jet, Bulletin of JSME, vol. 27, no. 230, pp. 1709-1715, 1984.

Skrebkov, G.P., Turbulent Pulsations in a Liquid Jet, and Its Atomization, Journal of Applied Mechanics and Technical Physics (Foreign Technology Division), no. 3, pp. 142-151, 1966.

Sterling, A.M. and Abbott, W.T., Mechanisms of water jet instability, Proceedings of the First U.S. Water Jet Symposium, Colorado School of Mines Press, Golden, CO, pp. I-4.1 to I-4.6, 1981.

Sterling, A.M. and Sleicher, C.A., The instability of capillary jets, Journal of Fluid Mechanics, vol. 68, no. 3, pp. 477-495, 1975.

Tafreshi, H.V. and Pourdeyhimi, B., The effects of nozzle geometry on waterjet breakup at high Reynolds numbers, Experiments in Fluids, vol. 35, no. 4, pp. 364-371, 2003. 
Tang, L. and Masutani, S.M., Laminar to Turbulent Flow Liquid-liquid Jet Instability and Breakup, The Thirteenth International Offshore and Polar Engineering Conference, International Society of Offshore and Polar Engineers, pp. 317-324, 2003.

Tate, T., On the magnitude of a drop of liquid formed under different circumstances, The London, Edinburgh, and Dublin Philosophical Magazine and Journal of Science, vol. 27, no. 181, pp. 176-180, 1864.

Tonkonogiy, Y.L., Buz, V.N., Garbuz, A.A., and Kalinchak, A.I., Effect of roughness on the transition Reynolds number, Fluid mechanics: Soviet research, vol. 19, no. 4, pp. 13-19, 1990.

Torda, T.P., Evaporation of drops and breakup of sprays, Astronautica Acta, vol. 18, pp. 383-393, 1973.

Trettel, B., Estimating turbulent kinetic energy and dissipation from internal flow loss coefficients, ICLASS 2018, Herrmann, M. and Desjardins, O. (Eds.), Chicago, IL, 2018, Paper no. 328.

Trettel, B., Improving the validation of turbulent jet breakup models, ILASS-Americas 2019, Owkes, M. (Ed.), Tempe, AZ, 2019, Paper no. 18.

Trettel, B., Conditional damped random surface velocity theory of turbulent jet breakup, Atomization and Sprays, vol. 30, 2020a.

Trettel, B., 2020b. pipe-jet-breakup-data, accessed February 19, 2020, from https://github.com/btrettel/ pipe-jet-breakup-data

Trettel, B., Turbulent Jet Breakup: Theory and Data, PhD dissertation, University of Texas, Austin, TX, 2020c.

Trujillo, M.F., Gurjar, S., Mason, M., and Agarwal, A., Global characterization of the spray formation process, Atomization and Sprays, vol. 28, no. 9, pp. 811-835, 2018.

van de Sande, E. and Smith, J.M., Jet break-up and air entrainment by low velocity turbulent water jets, Chemical Engineering Science, vol. 31, no. 3, pp. 219-224, 1976.

Vliem, H., The Influence of Restrictions on the Breakup Pattern of Turbulent Water Jets; a Simple Model of the External Urinary Stream of the Human Male, MS thesis, Technische Hogeschool Delft, Delft, Netherlands, 1975.

von Ohnesorge, W., The formation of drops by nozzles and the breakup of liquid jets, Translation, University of Texas at Austin, 2019.

Weber, C., Breakup of a liquid jet, Translation, University of Texas at Austin, 2019.

Wu, K.J., Su, C.C., Steinberger, R.L., Santavicca, D.A., and Bracco, F.V., Measurements of the Spray Angle of Atomizing Jets, Journal of Fluids Engineering, vol. 105, no. 4, pp. 406-413, 1983.

Wu, P.K., Liquid Surface Breakup of Nonturbulent and Turbulent Liquids, PhD dissertation, University of Michigan, Ann Arbor, MI, 1992.

Wu, P.K. and Faeth, G.M., Aerodynamic effects on primary breakup of turbulent liquids, Atomization and Sprays, vol. 3, no. 3, pp. 265-289, 1993.

$\mathrm{Wu}$, P.K. and Faeth, G.M., Onset and end of drop formation along the surface of turbulent liquid jets in still gases, Physics of Fluids, vol. 7, no. 11, pp. 2915-2917, 1995.

Wu, P.K., Miranda, R.F., and Faeth, G.M., Effects of initial flow conditions on primary breakup of nonturbulent and turbulent round liquid jets, Atomization and Sprays, vol. 5, no. 2, pp. 175-196, 1995.

Wu, P.K., Tseng, L.K., and Faeth, G.M., Primary breakup in gas/liquid mixing layers for turbulent liquids, Atomization and Sprays, vol. 2, no. 3, pp. 295-317, 1992.

Volume 30, Issue x, 2020 